Research Article

\title{
Study of Zuojin Pill in Treating Chronic Atrophic Gastritis by UPLC-Q-TOF/MS Based on Serum and Urine Metabolomics Combined with Network Pharmacology
}

\author{
Shihua Wu $\mathbb{D}^{1,2}$ Xing Chen, ${ }^{1,2}$ Honghong Liu, ${ }^{3}$ Ruilin Wang, ${ }^{3}$ Jianyu Li, ${ }^{3}$ Jianxia Wen, ${ }^{1,2}$ \\ Tao Yang, ${ }^{1,2}$ Ying Wei, ${ }^{1,2}$ Sichen Ren, ${ }^{1,2}$ Shizhang Wei, ${ }^{1,2}$ Manyi Jing, ${ }^{2}$ Haotian Li, ${ }^{2}$ \\ Min Wang, ${ }^{2}$ Houlin Xia $\mathbb{1 0}^{1},{ }^{1}$ and Yanling Zhao $\mathbb{1}^{2}$ \\ ${ }^{1}$ College of Pharmacy, Chengdu University of Traditional Chinese Medicine, Chengdu, China \\ ${ }^{2}$ Department of Pharmacy, The Fifth Medical Center of PLA General Hospital, Beijing, China \\ ${ }^{3}$ Integrative Medical Center, The Fifth Medical Center of PLA General Hospital, Beijing, China \\ Correspondence should be addressed to Houlin Xia; smile170264@163.com and Yanling Zhao; zhaoyl28552855@163.com
}

Received 2 November 2020; Revised 6 February 2021; Accepted 19 February 2021; Published 1 March 2021

Academic Editor: Mohamed Abdel-Rehim

Copyright (c) 2021 Shihua Wu et al. This is an open access article distributed under the Creative Commons Attribution License, which permits unrestricted use, distribution, and reproduction in any medium, provided the original work is properly cited.

\begin{abstract}
Zuojin Pill (ZJP) is widely used for the treatment of gastrointestinal diseases, while its specific mechanism has not been systematically investigated. The aim of this study was to explore the therapeutic effects and potential mechanism of ZJP in chronic atrophic gastritis (CAG) through UPLC-Q-TOF/MS-based metabolomics combined with network pharmacology. ZJP and omeprazole significantly reduce contents of IL-1 $\beta$, IL-6, IL-10, and iNOS and improve pathological characteristics. Metabolomic results indicated that the therapeutic effects of ZJP were mainly related to ten metabolites, namely, choline, L-threonine, hydroxypyruvic acid, creatine, taurine, succinic acid, cis-aconitic acid, citric acid, succinic acid semialdehyde, and uric acid. Pathway analysis showed that the treatment of CAG by ZJP was associated with taurine and hypotaurine metabolism; glyoxylate and dicarboxylate metabolism; glycine, serine, and threonine metabolism; glycerophospholipid metabolism; citrate cycle (TCA cycle), alanine, aspartate, and glutamate metabolism; butanoate metabolism; and purine metabolism. Validation of metabolic markers and key targets of network pharmacology through RT-PCR analysis showed that ZJP significantly downregulated a series of inflammatory markers, such as MAPK1, PKIA, RB1, SCN5A, RXRA, E2F1, PTGS1, IGF2, ADRB1, ADRA1B, PTGS2, and GABRA1. This study was the first to use a combination of metabolomics and network pharmacology to clarify the therapeutic effects of ZJP on CAG and the regulation of multiple metabolic pathways.
\end{abstract}

\section{Introduction}

In 1993, the World Health Organization's International Agency for Research and Cancer (IARC) defined Helicobacter pylori $(H$. pylori) as a type 1 carcinogen. Several studies have confirmed that $H$. pylori infection is a risk factor for the development of chronic atrophic gastritis (CAG) and even gastric cancer $[1,2]$. CAG is a common clinical disease of the digestive system and is typically characterized with thinning of the gastric mucosa epithelium and atrophy of the glands. Although triple and quadruple therapies are commonly used in clinical practice to treat $\mathrm{CAG}$, long-term therapy can enhance bacterial resistance, decrease eradication rates, and lead to serious side effects such as gastrointestinal reactions and liver dysfunction [3]. Therefore, there is an urgent clinical need for an effective drug with a few side effects to treat $H$. pylori-induced CAG.

Zuojin Pill (ZJP) is a famous Chinese herbal formula which was first recorded in an ancient medical treatise "Danxi New Law" several hundreds of years ago. ZJP consists of two traditional Chinese medicines named Coptidis Rhizoma (CR) and Euodiae Fructus (EF) in a weight ratio of $6: 1(\mathrm{w} / \mathrm{w})$ and has been officially listed in the Chinese Pharmacopoeia. The main ingredients of CR are 
alkaloids, including berberine, palmatine, coptisine, and epiberberine. Studies have shown that CR has a wide range of pharmacological effects, such as antibacterial, antiatherosclerotic, antiviral, antioxidant, antihepatic steatosis, anti-inflammatory, and antitumor effects [4]. The main chemical constituents of $\mathrm{EF}$ also include alkaloids and terpenes which have antibacterial, hypotensive, and antihypoxic pharmacological activities. ZJP has a variety of pharmacological effects in treating gastrointestinal disorders such as gastritis, cholecystitis, and peptic ulcers through being anti-inflammatory, antiulcer, and antiacid and inhibits the growth and proliferation of $H$. pylori $[5,6]$. Previous studies have shown that ZJP and its major components exhibit multiple pharmacological effects against cancer through multiple mechanisms [7, 8]. ZJP exerts a gastroprotective role by modulating the NF- $\mathrm{KB}$ signaling pathway and inhibiting inflammatory cytokines expression, thereby reducing the risk of gastric ulcers [9]. ZJP treats gastrointestinal diseases by restoring the rhythm of the stomach as well as improving gastrointestinal motility [10]. The ethanolic extract of ZJP inhibited inflammation by decreasing the expression of iNOS, COX-2, IL-6, IL- $1 \beta$, and TNF- $\alpha$ [11]. However, the in-depth mechanism of ZJP in treating CAG has not been fully elucidated.

Metabolomics, an important branch of systems biology, provides a systematic study of the relationships between drugs, diseases, and metabolites. Metabolomics can characterize the overall physiological and pathological state after exogenous stimulation and also systematically identify endogenous small molecule metabolites. Modern analytical technologies include nuclear magnetic resonance (NMR), gas chromatography-mass spectrometry (GC-MS), and liquid chromatography-mass spectrometry (LC-MS), combined with multivariate data analysis, such as principal component analysis (PCA), partial least squares discriminate analysis (PLS-DA), and orthogonal projection to latent structures-discriminant analysis (OPLS-DA) [12]. Among these methods, ultrahigh performance liquid chromatography with quadrupole time-of-flight (UPLC-Q-TOF) has the advantages of high selectivity, resolution, and accuracy, which is particularly suitable for traditional Chinese medicine (TCM) [13]. Due to the easy collection of blood and urine samples and being closely related to diseases, choosing these two biological fluids is an effective method for metabolomics research [14]. Serum metabolite profiles are considered to be important indicators reflecting physiology and pathology and help to understand the metabolites, metabolic pathways, and mechanisms of the disease. Urine metabolite profiles are important for discovering disease biomarkers, especially excreted metabolites. Serum samples usually represent low-polarity metabolites, while urine samples consist of high-polarity metabolites. These two metabolite profiles complement each other; reflect overall characteristics; and demonstrate disease mechanisms, interventions, and metabolic pathways. [15]. Nevertheless, there is a large variety of metabolites and a wide range of concentrations, and the use of metabolomics alone to analyze the mechanisms of TCM has limitations. In recent years, network pharmacology has been used to study and predict biological metabolism and the interaction between TCM and disease targets [16]. Network pharmacology provides a systematic method to understand the interaction between disease characteristics and drug targets [17]. A growing number of studies have shown that network pharmacology, as an effective tool to study the overall role of TCM, can better understand the complexity of TCM and clarify the potential mechanisms through the active chemical components and therapeutic targets of TCM [18]. In addition, metabolomics combined with network pharmacology has been successfully used to explore the interactions between TCM and disease targets, providing an important direction for the study of therapeutic mechanisms of TCM.

In order to clarify the therapeutic effects and mechanism of ZJP on CAG, this study explored the pathological changes and serum biochemical indicators of ZJP through basic pharmacological experiments. A rat model of CAG was established by $H$. pylori gavage, and the success of model establishment was assessed by a rapid urease kit. In this study, UPLC-Q-TOF/MS serum metabolomics and urine metabolomics were used to determine the changes of endogenous metabolites, combined with network pharmacology to screen relevant targets and then to clarify possible biomarkers and potential metabolic pathways for ZJP treatment of CAG.

\section{Experimental}

2.1. Materials and Instruments. Rat interleukin-6 (IL-6, Lot: 202007), interleukin-10 (IL-10, Lot: 202007), interleukin-1 $\beta$ (IL-1 $\beta$, Lot: 202007), and inducible nitric oxide synthase detection (iNOS, Lot: 202007) ELISA kits were purchased from Shanghai MLBIO Biotechnology Co. Ltd. (Shanghai, China). RNA-Quick Extraction Kit (No:RN001) was purchased from Beijing Yishan Biotechnology Co. Ltd. (Beijing, China).

2.2. Preparation of ZJP. CR (Lot: 18011901) and EF (Lot: 17021602) were purchased from Beijing Lvye Pharmaceutical Co., Ltd. (Beijing, China). Firstly, ZJP was extracted with boiling water $(1 / 10, \mathrm{w} / \mathrm{w})$ two times, $1.0 \mathrm{~h}$ each time. The aqueous extract of ZJP was then dried to powder in a freeze-vacuum drying oven, and the final mass ratio of ZJP was approximately $25.59 \%$.

2.3. Bacterial Culture and Animal Handling. H. pylori strain (strain number: ICDC111001) was supplied by Prof. Jianzhong Zhang (Chinese Disease Control and Prevention Center, Beijing, China). H. pylori strains cultured on blood agar (Thermo Fisher Scientific, China) in a 3-5 days cycle.

Thirty-six male Sprague-Dawley rats of $170-190 \mathrm{~g}$ were purchased from Beijing Sibeifu Animal Breeding Center [Permission No. SCXK-(Jing) 2016-0002] and fed in a standard environment (temperature: $25^{\circ} \mathrm{C} \pm 2^{\circ} \mathrm{C}$, humidity: $55 \% \pm 5 \%, 12: 12 \mathrm{~h}$ light: dark cycle) at the Animal Experimental Center of the Fifth Medical Center of the PLA General Hospital. Rats in the control group were given saline by gavage, and rats in the model groups were gavaged with 
H. pylori suspension $\left(1.5 \times 10^{8}\right.$ colony forming units (CFU)/ $\mathrm{ml}, 1.5 \mathrm{~mL}$ ) four times a week for 8 weeks. After the successful establishment of the CAG model, the model group rats were randomly divided into five groups. Then, except for the control group and the model group, ZJP $(0.63,1.26$, $2.52 \mathrm{~g} / \mathrm{kg} /$ day $)$ and omeprazole $(1.8 \mathrm{mg} / \mathrm{kg} /$ day, as a positive group) groups were administered by gavage for 4 weeks. Urine was collected from rats for 12 hours after the last administration, and then the rats were anesthetized by intraperitoneal injection of $20 \%$ urethane. Then, the stomach, blood, and urine were collected. The blood was centrifuged at 3,500 rpm for $15 \mathrm{~min}$ and serum was separated. The urine, serum, and stomach tissues were stored at $-80^{\circ} \mathrm{C}$ for further experiments.

Serum levels of IL-6, IL-10, IL-1 $\beta$, and iNOS were measured according to the instructions of ELISA kit. Stomach tissues were fixed in $10 \%$ neutral formalin buffer for 24 hours. The fixed gastric tissues were embedded in paraffin wax and then cut into slices using a slicer. Hematoxylin-eosin (HE) was used to highlight gastric sinus injury and inflammatory cell infiltration.

\subsection{Sample Preparation and UPLC-Q-TOF/MS Testing. A} mixture of $600 \mu \mathrm{L}$ of methanol with $200 \mu \mathrm{L}$ of serum was allowed to stand for $20 \mathrm{~min}$ at $4^{\circ} \mathrm{C}$ and then centrifuged at $12,000 \mathrm{rpm}$ for $10 \mathrm{~min}$. Next, the supernatant was aspirated and filtered through a $0.22 \mu \mathrm{m}$ micropore filter, and the filtrate was collected and analyzed in the next step. The urine sample was prepared in the same procedure as the serum sample.

The analysis of serum metabolic profile and urine metabolic profile was performed by Agilent 6550 iFunnel Q-TOF LC/MS (Agilent Technologies, USA) system. On a ZORBOX RRHD $\mathrm{C}_{18}$ analytical column (2.1 mm i.d. $\times 100 \mathrm{~mm}, 1.8 \mu \mathrm{mi}$ d., Agilent Technologies, USA), $4 \mu \mathrm{L}$ of each sample was taken and injected into the system, and samples were separated at $30^{\circ} \mathrm{C}$. Solvent I (water containing $0.1 \%$ formic acid) and solvent II (acetonitrile containing $0.1 \%$ formic acid) were used as the mobile phase and separated at a flow rate of $0.30 \mathrm{~mL} / \mathrm{min}$ for $25 \mathrm{~min}$ with a linear gradient of $100 \%$ I over $0-1.0 \mathrm{~min}$, over $100-60 \%$ I over $1.0-9.0 \mathrm{~min}, 60-10 \%$ I over $9.0-19.0 \mathrm{~min}, 10-0 \%$ I over $19.0-21.0 \mathrm{~min}$, and $100 \% \mathrm{II}$ over $21.0-25.0 \mathrm{~min}$.

Positive and negative mode electrospray source parameters were set as follows: electrospray capillary voltage of $3.5 \mathrm{kV}$ in negative mode and $4 \mathrm{kV}$ in positive mode, mass range of $\mathrm{m} / \mathrm{z} 50-1200$, gas temperature and flow rate of $225^{\circ} \mathrm{C}$ and $13 \mathrm{~L} / \mathrm{min}$, atomizer setting of $20 \mathrm{psi}$, and sheath gas temperature and flow rate of $275^{\circ} \mathrm{C}$ and $12 \mathrm{~L} / \mathrm{min}$ with a nozzle voltage of $2000 \mathrm{~V}$ in both positive and negative ion modes.

2.5. Data Extraction and Multivariate Analysis. Data extraction, peaks detection, and comparison were performed through MassHunter Profinder software (Agilent, CA, USA). The full scan mode was applied to the mass range $\mathrm{m} / \mathrm{z}$ 80-1000 and set the initial and final retention times for data collection. Data were standardized using the MetaboAnalyst website (https://www.metaboanalyst.ca) and then analyzed by principal component analysis (PCA) and orthogonalpartial least squares discriminant analysis (OPLS-DA) using SIMCA-P 14.1 software (Umetrics, Umea, Sweden).

2.6. Potential Biomarker Identification and Pathway Enrichment Analysis. Biomarkers were identified by metabolic differential metabolite screening. In the OPLS-DA analysis, the screening conditions for differential metabolites were set to VIP $>1.0, \mid P($ corr $) \mid \geq 0.58, P<0.05$, and the obtained metabolites were identified as potential biomarkers [19]. Metabolites were identified based on the precise molecular weight of the Human Metabolome Database (HMDB) (molecular weight error $<20 \mathrm{ppm}$ ). The identified compounds were resubmitted to MetaboAnalyst for the enrichment of potential signaling pathways.

2.7. ZJP Drug Target Identification and Network Pharmacology Analysis. The chemical composition and associated targets of CR and EF were collected in the TCMSP database (https://tcmspw.com/tcmsp.php). Potential metabolite-associated protein targets were collected via the MBROLE 2.0 database (http://csbg.cnb.csic.es/mbrole2/index.php). The GeneCards database (https://www.genecards.org/) was used to collect CAG-related protein targets. Uniprot ID was used to convert different types of protein IDs, and then the protein interaction information was used to build a network of component-metabolite-target interactions. Finally, the component-metabolite-target interaction network was visualized and analyzed through Cytoscape 3.7.1 software.

2.8. Real-Time Polymerase Chain Reaction (RT-PCR) Detection. Total RNA was extracted from gastric tissues using the RNA-Quick Extraction Kit and reverse transcribed to cDNA using the PrimerScript RT kit (Promega, Madison, USA). The cDNA was subsequently PCR amplified by ABI Step One Plus. The data were analyzed by the $2^{-\Delta \Delta C T}$ method, and the information of primers is listed in Table 1.

2.9. Statistical Analysis. All data were presented as mean \pm standard deviation (SD) and analyzed using the SPSS software program (version 25.0; SPSS Inc., Chicago, IL, USA). The differences were considered to be statistically significant when $P<0.05$ and highly significant when $P<0.01$.

\section{Results}

3.1. ZJP Reduces H. pylori-Induced CAG Pathological Damage and Serum Inflammatory Factors. As shown in Figure 1, the rats in the control group had normal gastric histology, and the rats in the model group showed pathological features of CAG, such as ruptured epithelial cells, atrophy and misarrangement of glands, and inflammatory cell infiltration. The ZJP and omeprazole groups were effective in improving gastric mucosal lesions, glandular loss, and reducing inflammatory infiltration. Moreover, as shown in Figure 2, the 
Table 1: Primers used for real-time PCR.

\begin{tabular}{lcc}
\hline Primers & Sequence-forward & Sequence-reverse \\
\hline PTGS1 & TCTGGACCTGGCTTCGGAGTTC & TGGACCGCACCGTGAGTACC \\
PTGS2 & AGGTCATCGGTGGAGAGGTGTATC & CGGCACCAGACCAAAGACTTCC \\
MAPK1 & TGAAGACACAGCACCTCAGCAATG & GGTGTTCAGCAGGAGGTTGGAAG \\
GABRA1 & AGTGCCAGAAATTCCTCCCAAAG & CAATCAGAGCCGAGAACACGAAGG \\
IGF2 & CAGCCGTGGCATCGTGGAAG & CGGGGTATCTGGGGAAGTCGTC \\
PKIA & TTCCTCTGCAAGTGGCAACAGC & TGCTTCTCCCTGGGCTTCGC \\
SCN5A & GGAAGGTCTGGTGGCGATTGC & TTCCGCTCCTCCAGGTAGATGTC \\
E2F1 & TGGTGGCTGCTGACTCACTCC & TGGGGTGGGGAAAGGCTGATG \\
ADRB1 & CTCATCGTGCTGCTCATCGTAGTG \\
RB1 & CAAGCAGCCTCAGCCTTCCATAC \\
ADRA1B & ATCGTGGGCAACATCTTGGTCATC & GCCAGGGACATGATGAAGAGGTTG \\
RXRA & ACACTGGGCTTCGGGACTGG & TGTGTTCTGGCTCTGGGTGGTC \\
-Actin & CCCGCGAGTACAACCTTCTTG & ACTCAACAGCAGGTCAGCAATGG \\
\hline
\end{tabular}

serum levels of IL-1 $\beta$, IL-6, IL-10, and iNOS were significantly increased in the model group compared with the control group $(P<0.01)$. The serum contents of IL- $1 \beta$, IL-6, IL-10, and iNOS were decreased after ZJP and omeprazole administration. In particular, IL-1 $\beta$, IL-6, IL-10, and iNOS levels were significantly reduced after administration of $2.52 \mathrm{~g} / \mathrm{kg}$ ZJP and omeprazole compared to the model group $(P<0.05, P<0.01)$.

3.2. Multivariate Data Analysis. PCA and OPLS-DA are powerful statistical tools, which can identify the separation between different groups based on the results of different spectra [20]. After Pareto normalization of data from the control group, model group, and $2.52 \mathrm{~g} / \mathrm{kg}$ ZJP group, the three groups were distinguished by PCA using SIMCA-P 12.0 software. As shown in Figure 3, the control group, the model group, and $2.52 \mathrm{~g} / \mathrm{kg}$ ZJP group showed good separation in both ESI+ and ESI- modes, indicating that the metabolic distribution of the three groups was significantly different. As shown in Figures 3(b) and 3(d), there was a clear separation between the control group and the model group in ESI- mode, and the $2.52 \mathrm{~g} / \mathrm{kg}$ ZJP group was located between the control group and the model group, indicating that ZJP has a regulatory effect with metabolic abnormalities. The ESI+ model has a poor trend in the $2.52 \mathrm{~g} / \mathrm{kg}$ ZJP group; therefore, further analysis is required (Figures 3(a) and 3(c)).

Then, the OPLS-DA analysis was established for the ESI+ and ESI- modes of serum and urine samples. Given that OPLS-DA can only be used to screen the differentially expressed metabolites between the two groups [21], we identified the differential metabolites by OPLS-DA analysis for the control group and the model group, and the model group and $2.52 \mathrm{~g} / \mathrm{kg}$ ZJP group, respectively. As shown in Figures 4 and 5, the OPLS-DA mode established by the control group and model group data for ESI+ and ESImodes could explain the 0.999, 0.993, 0.991, and 0.999 variance of the response variable $\left(R^{2} Y\right)$ and the cumulative explained variance for modeling in cross-validations $\left(Q^{2}\right)$ was $0.975,0.938,0.857$, and 0.92 , respectively. For serum and urine data of the model group versus $2.52 \mathrm{~g} / \mathrm{kg}$ ZJP group in ESI+ and ESI- modes, $R^{2} Y$ values were $0.998,0.987,0.98$, and 0.989 with $Q^{2}$ values being $0.979,0.81,0.774$, and 0.91 , respectively. These parameters indicated that the models have good explanatory and predictive capabilities. The S-plot was used to investigate the inherent clustering variables. In the S-plot, variables with an average VIP value above 1 and an absolute $P$ (corr) value above 0.58 can be considered as potential biomarkers. The S-plot shows significant differences in endogenous metabolites between the control, model, and $2.52 \mathrm{~g} / \mathrm{kg}$ ZJP groups. The variables located at the ends of the S-plot made an important contribution to the separation of the two groups. The predictive capability of the established OPLS-DA analysis is then verified by a permutation test $(n=100)$. The results showed that the $R^{2}$ and $Q^{2}$ values of serum and urine data were lower than the permutation tests, indicating that the OPLS-DA analysis has a superior fit and better predictive ability.

\subsection{Identification of Potential Metabolites in ZJP Treatment.} For more accurate identification, potential metabolites were selected based on $|\mathrm{VIP}|>1$ and $\mid P($ corr $) \mid \geq 0.58$ in the S-plot extracted between the control group and the model group, and the model group and the $2.52 \mathrm{~g} / \mathrm{kg}$ ZJP group. At this threshold, multivariate and univariate analyses were performed using ANOVA analysis and $t$-test, respectively, to screen out variables that were significantly different. The $\mathrm{m} / \mathrm{z}$ of candidate metabolites were matched against online databases (including HMDB database and MetaboAnalyst database) to identify candidate biomarkers with significant changes. The mass tolerance value and the exact mass of the measured $\mathrm{m} / \mathrm{z}$ are defined as less than $20 \mathrm{ppm}$.

For serum samples, a total of six endogenous metabolites were screened in ESI+ mode and ESI- mode (see Table 2 for details). For urine samples, four endogenous metabolites were identified based on the above procedure (please see Table 2). These ten potential biomarkers are choline (C00114), L-threonine (C00188), hydroxypyruvic acid (C00168), creatine (C00300), taurine (C00245), succinic acid (C00042), cis-aconitic acid (C00417), citric acid (C00158), succinic acid semialdehyde (C00232), and uric acid (C00366). The levels of L-threonine, hydroxypyruvic acid, taurine, succinic acid, and cis-aconitic acid were significantly lower in the model group compared with the control group 


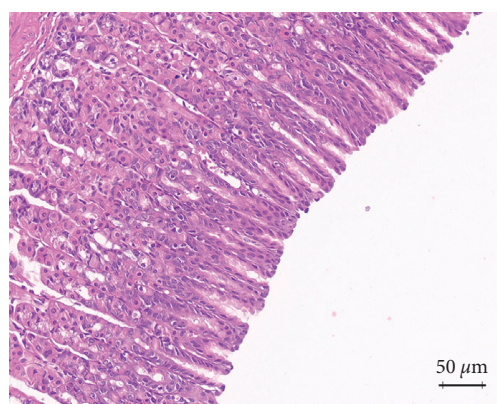

(a)

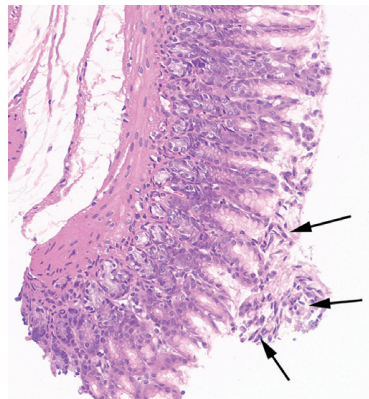

(d)

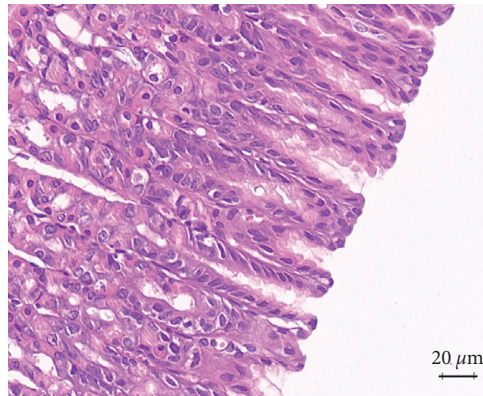

(g)

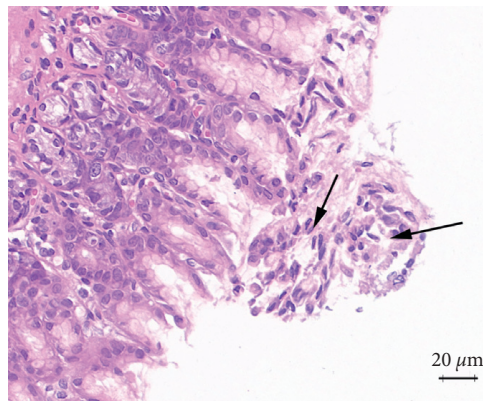

(j)

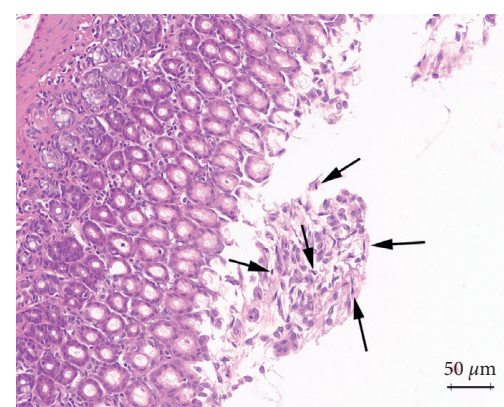

(b)

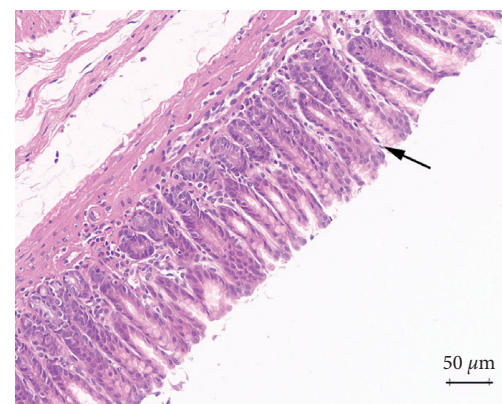

(e)

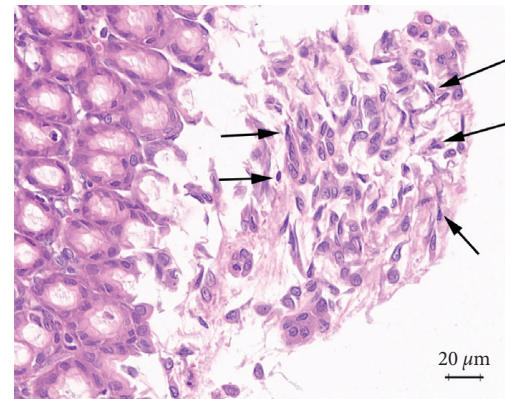

(h)

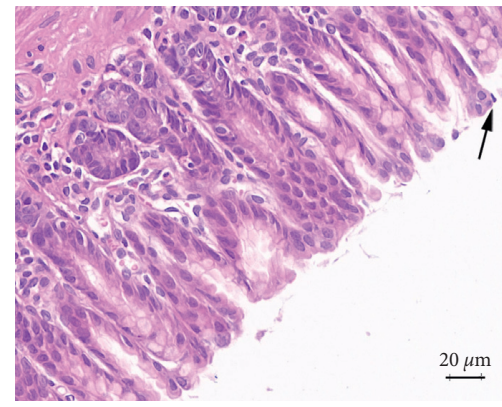

(k)

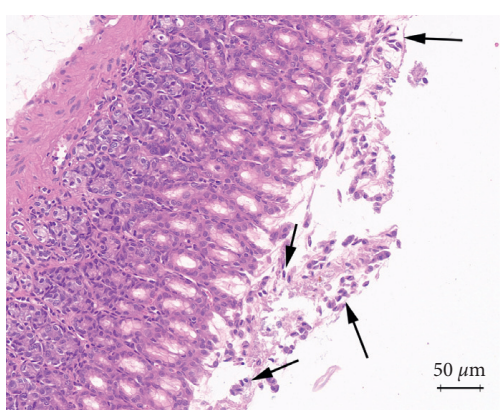

(c)

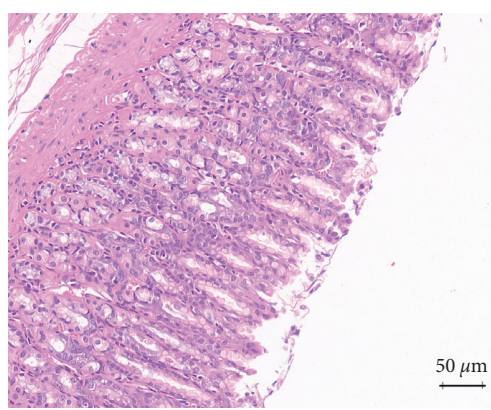

(f)

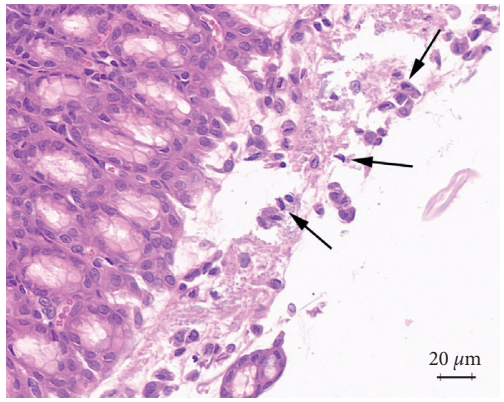

(i)

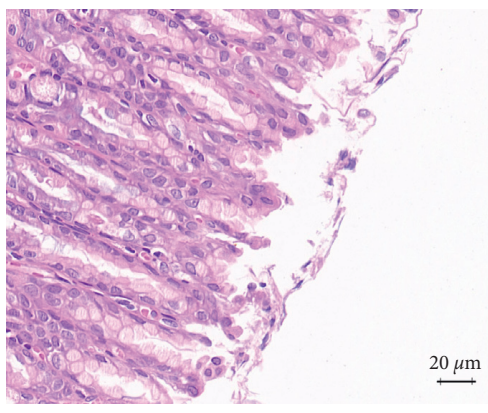

(l)

Figure 1: ZJP affects gastric histopathology in CAG rats. (a) Control group. (b) Model group. (c) $0.63 \mathrm{~g} / \mathrm{kg}$ ZJP group. (d) $1.26 \mathrm{~g} / \mathrm{kg}$ ZJP group. (e) $2.52 \mathrm{~g} / \mathrm{kg}$ ZJP group. (f) Omeprazole group (HE staining, $\times 200$ magnification). (g) Control group. (h) Model group. (i) $0.63 \mathrm{~g} / \mathrm{kg}$ ZJP group. (j) $1.26 \mathrm{~g} / \mathrm{kg}$ ZJP group. (k) $2.52 \mathrm{~g} / \mathrm{kg}$ ZJP group. (l) Omeprazole group (HE staining, $\times 400$ magnification). 


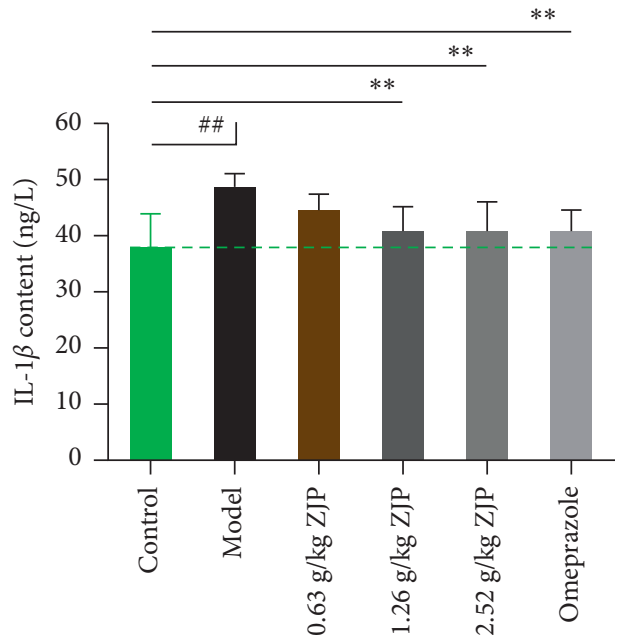

(a)

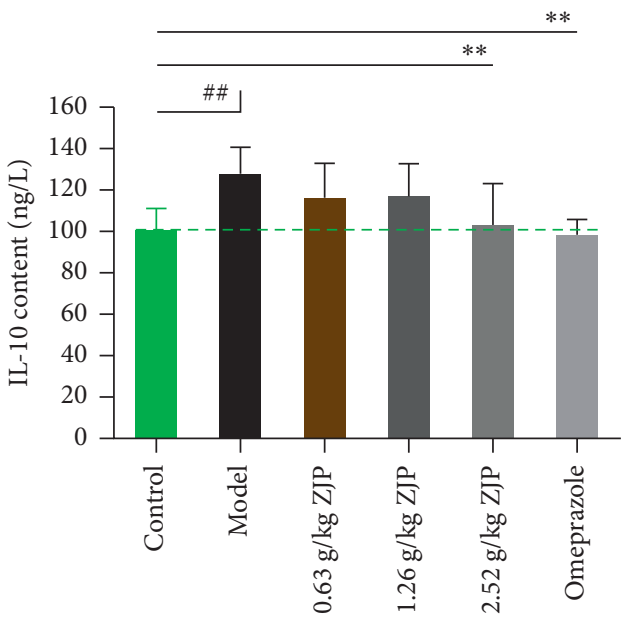

(c)

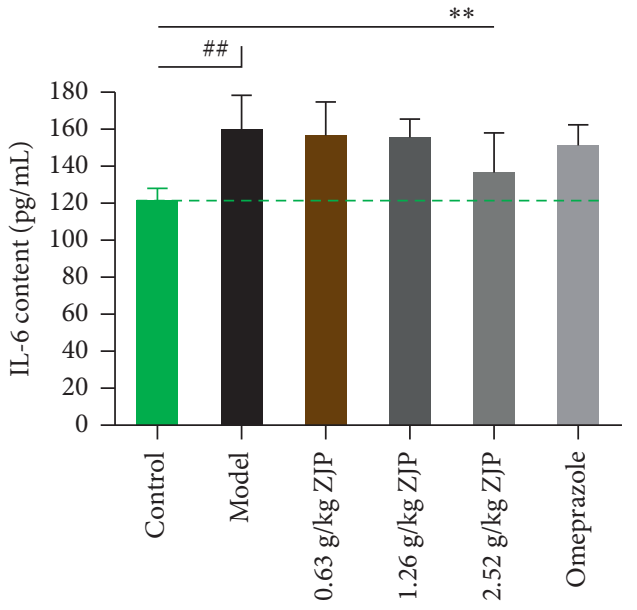

(b)

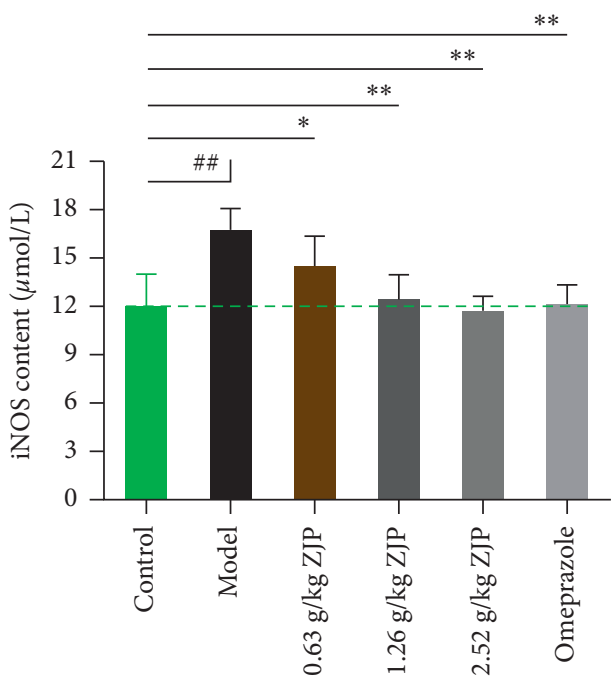

(d)

Figure 2: ZJP affects serum biochemical parameters (mean \pm SD, $n=6$ ). (a) IL- $1 \beta$ content. (b) IL-6 content. (c) IL-10 content. (d) iNOS content. ${ }^{\#} P<0.01$, compared with the control group. ${ }^{*} P<0.05$ and ${ }^{* *} P<0.01$, compared with the model group.

$(P<0.05$ and $P<0.01)$. In addition, the levels of choline, creatine, citric acid, succinic acid semialdehyde, and uric acid were significantly higher in the model group compared with the control group $(P<0.01)$. The mean peak area of these metabolites tended to be normalized in the $2.52 \mathrm{~g} / \mathrm{kg}$ ZJP group compared with the model group $(P<0.05$, $P<0.01$ ), showing good therapeutic efficacy. The mean peak area of these metabolites is shown in Figure 6 to visualize the effects of ZJP. The alterations of these parameters indicated that the rats in the model group had metabolic abnormalities after gastric mucosal inflammatory injury, and the metabolic markers returned to normal levels after ZJP treatment.

3.4. Metabolic Pathway Analysis. To explore the possible mechanism of ZJP on CAG, potential metabolites identified from serum and urine samples were introduced into MetaboAnalyst to construct metabolic pathways, and twenty-eight metabolic pathways were obtained (Figure 7).
Seven of these 28 metabolic pathways play an important role, including Taurine and hypotaurine metabolism; glyoxylate and dicarboxylate metabolism; glycine, serine, and threonine metabolism; glycerophospholipid metabolism; citrate cycle (TCA cycle), alanine, aspartate, and glutamate metabolism; butanoate metabolism; and purine metabolism. The match status, $P$ value, $-\log (P)$, and impact of each pathway are listed in Table 3.

3.5. Network Pharmacology. In order to visualize the interaction between potential metabolites, disease targets, and ZJP chemical components, the components and potential disease targets were collected from the TCMSP database and GeneCards database, and combined with potential metabolites to construct a potential metabolite-CAG targetscomponent network, as shown in Figure 8. Potential metabolites were extended to relevant metabolic pathways through MBROLE for further exploring the relationship 


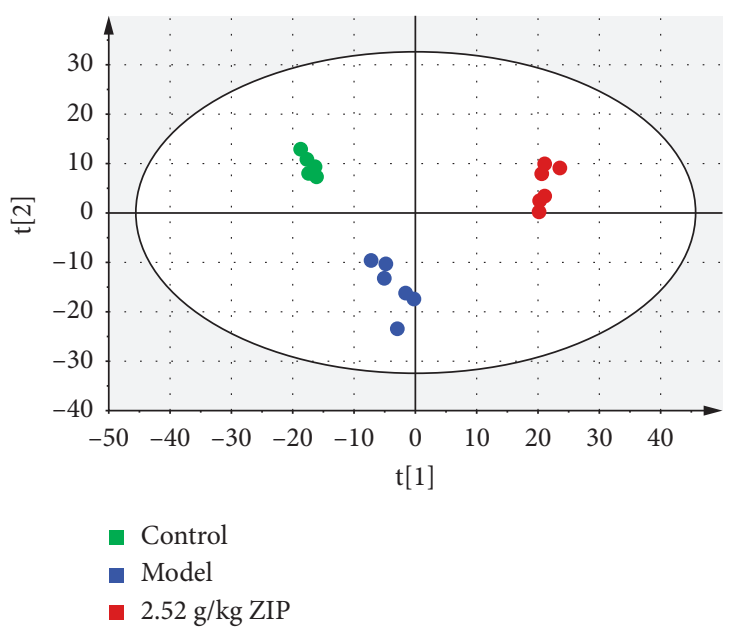

(a)

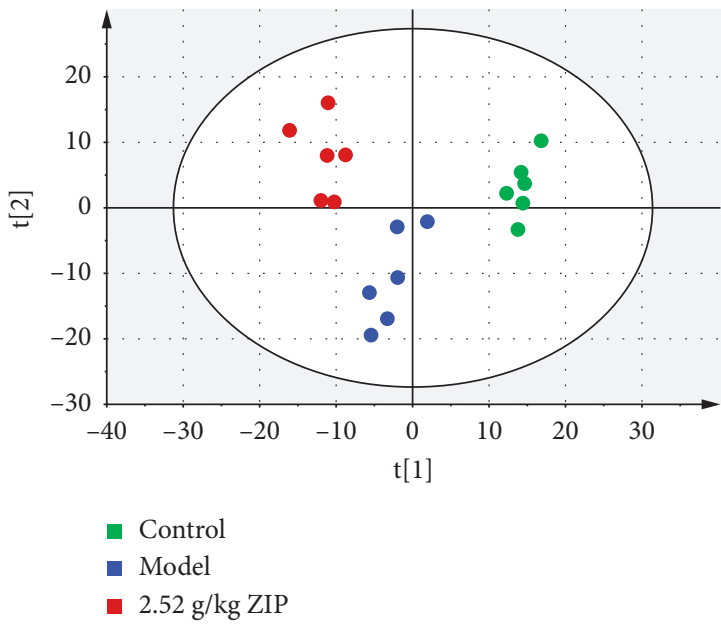

(c)

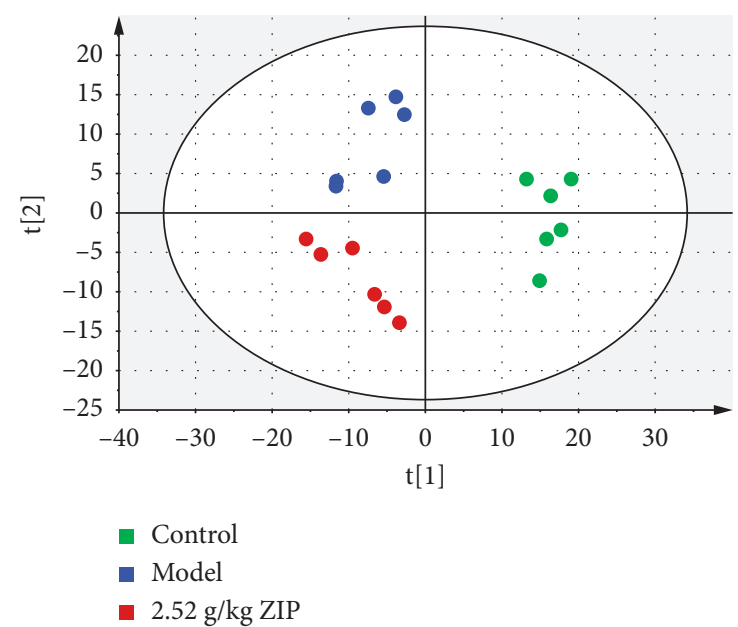

(b)

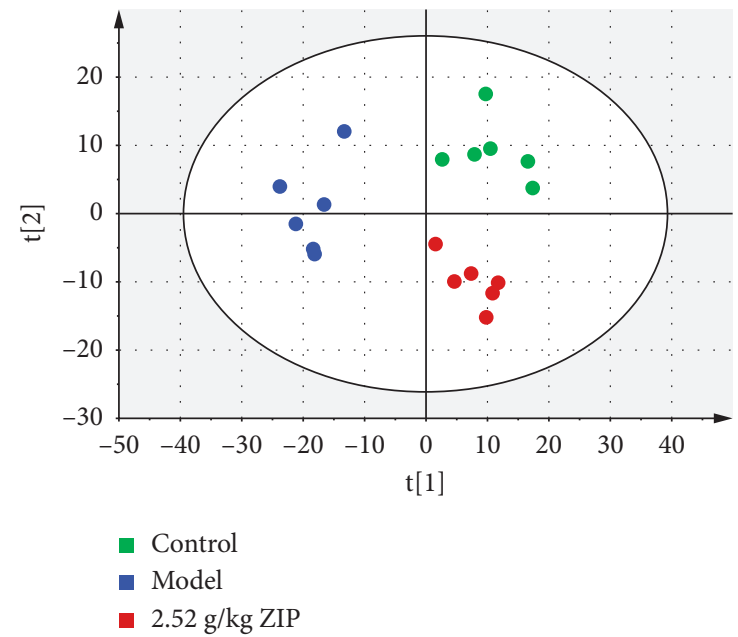

(d)

Figure 3: PCA score plot of the control group, the model group, and $2.52 \mathrm{~g} / \mathrm{kg}$ ZJP group. (a) ESI+ mode of PCA in serum samples between the three groups. (b) ESI- mode of PCA in serum samples between the three groups. (c) ESI+ mode of PCA in urine samples between the three groups. (d) ESI- mode of PCA in urine samples between the three groups.

between metabolite-related proteins and disease targets. The related information of 12 proteins in compound-disease target-metabolite is listed in Table 4. These proteins are directly regulated by 124 chemical components of ZJP (Figure 8(b)).

3.6. Effect of ZJP on the mRNA Expression in CAG Rats. CAG is a critical step in the development of gastric cancer, and the chance of cancer progressively increases as inflammation worsens. A large amount of clinical evidence shows that ZJP can inhibit gastric mucosal injury, neutrophil infiltration, and intestinal metaplasia in CAG patients. Thus, inflammation-related genes MAPK1, PKIA, RB1, SCN5A, RXRA, E2F1, PTGS1, IGF2, ADRB1, ADRA1B, PTGS2, and GABRA1 may play a more important role in the treatment of CAG by ZJP. To verify the validity of the network pharmacology predictions, RT-PCR was used to detect the mRNA expression of these genes in gastric tissues. As shown in Figure 9, the relative mRNA expression levels of MAPK1, PKIA, RB1, SCN5A, RXRA, E2F1, PTGS1, IGF2, ADRB1, ADRA1B, PTGS2, and GABRA1 were significantly elevated in the model group $(P<0.01)$. The expression levels of these genes in the ZJP groups were significantly decreased $(P<0.05$ and $P<0.01)$, indicating that ZJP was effective in treating CAG through anti-inflammatory treatment.

\section{Discussion}

Metabolomics analyzes the levels of small molecules in serum and urine to explore compounds with significant abnormalities and reveal mechanisms of disease development. Network pharmacology reveals the molecular mechanism of drug therapy by determining the interaction between chemical components and disease protein targets. The combination of metabolomics and network pharmacology can mine data extensively to determine the targets of metabolism and disease [22]. Therefore, in this study, serum 


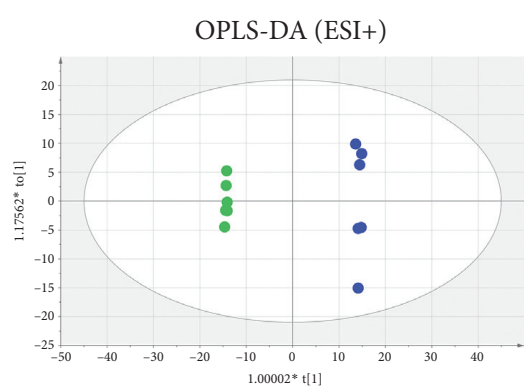

- Control

(a)

OPLS-DA (ESI+)

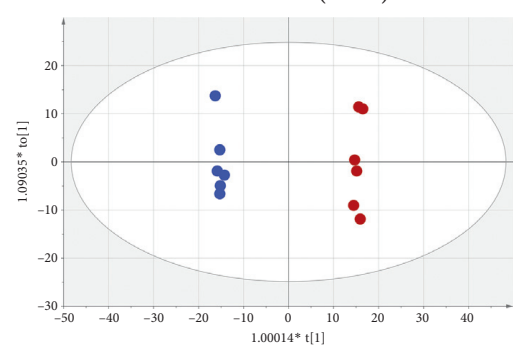

- Model $2.52 \mathrm{~g} / \mathrm{kgZIP}$

(d)

OPLS-DA (ESI-)

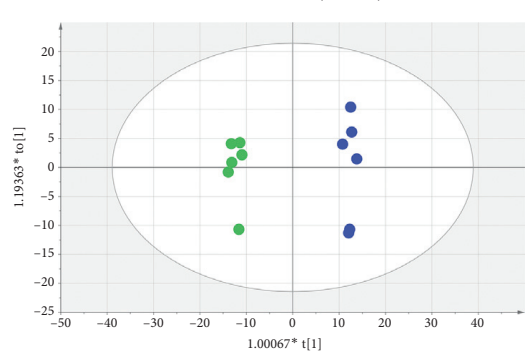

- Montrol

(g)

OPLS-DA (ESI-)

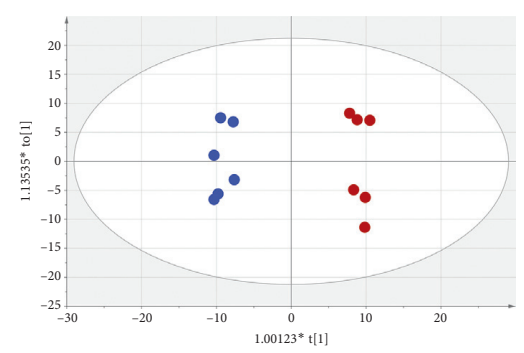

- $2.52 \mathrm{~g} / \mathrm{kg}$ ZJP

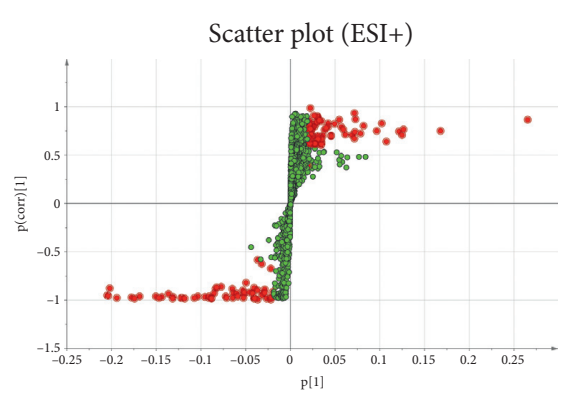

(b)

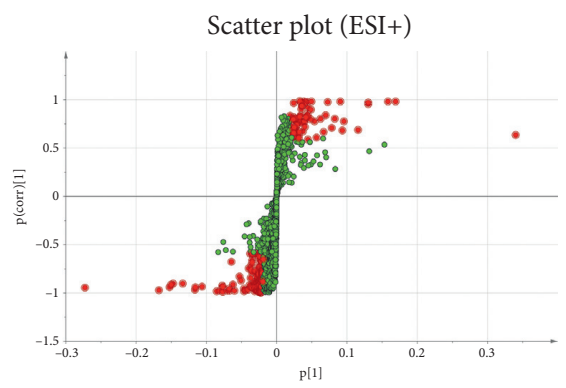

(e)

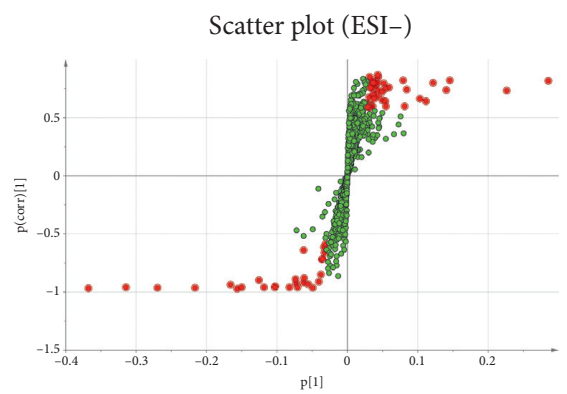

(h)

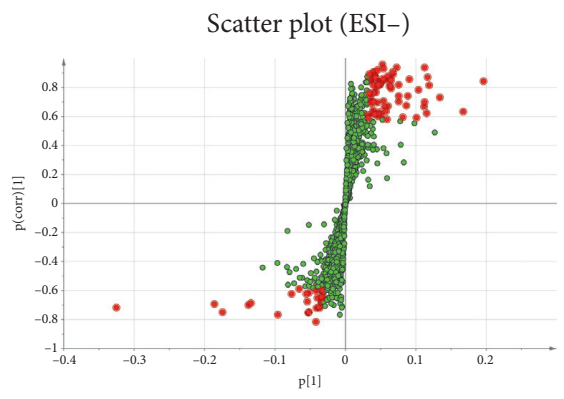

(k)

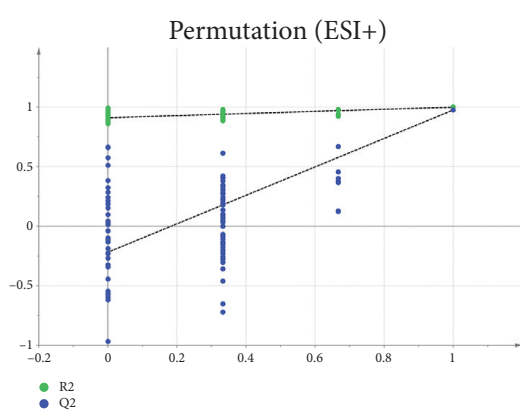

(c)

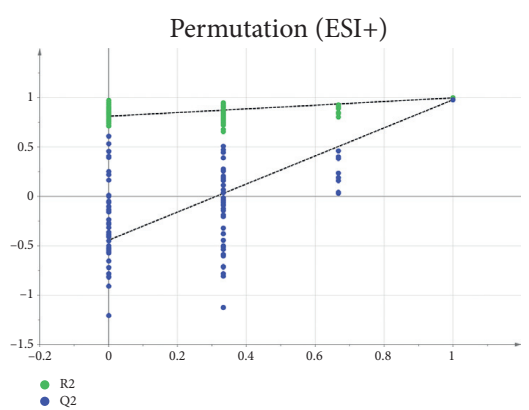

(f)

Permutation (ESI-)

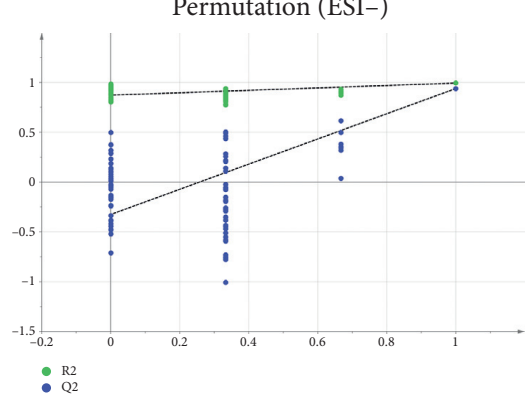

(i)

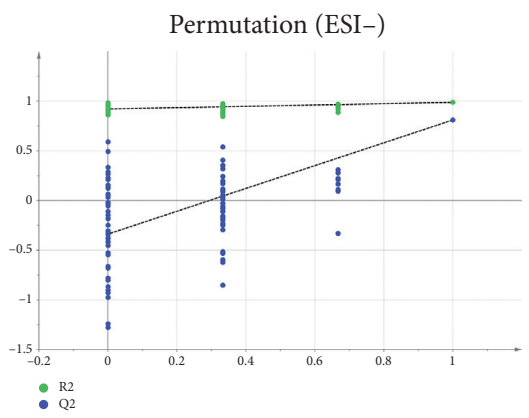

(1)

FIGURE 4: The OPLS-DA score plots, S-plot, and 100-permutation test generated of serum samples between the control group, model group, and $2.52 \mathrm{~g} / \mathrm{kg}$ ZJP group. OPLS-DA score plots were the pairwise comparisons in ESI+ mode, (a) control group versus model group and (d) model group versus $2.52 \mathrm{~g} / \mathrm{kg}$ ZJP group, as well as in ESI- mode, ( $\mathrm{g}$ ) control group versus model group and (j) model group versus $2.52 \mathrm{~g} / \mathrm{kg}$ ZJP group. S-plot of the OPLS-DA model in ESI+ mode, (b) control group versus model group and (e) model group versus $2.52 \mathrm{~g} / \mathrm{kg}$ ZJP group, as well as in ESI- mode, (h) control group versus model group and (k) model group versus $2.52 \mathrm{~g} / \mathrm{kg}$ ZJP group. (c) The 100 permutation test of the OPLS-DA model in ESI+ mode, control group versus model group and (f) model group versus $2.52 \mathrm{~g} / \mathrm{kg} \mathrm{ZJP}$ group, as well as in ESI- mode, (i) control group versus model group and (l) model group versus $2.52 \mathrm{~g} / \mathrm{kg}$ ZJP group. 


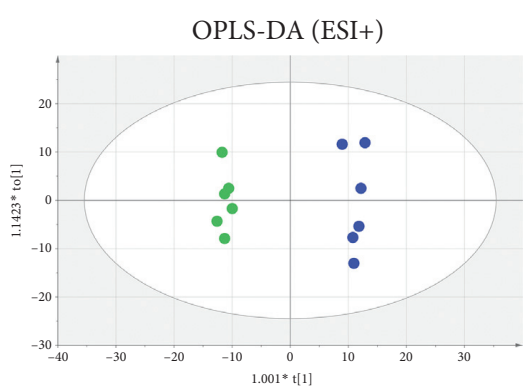

- Control

(a)

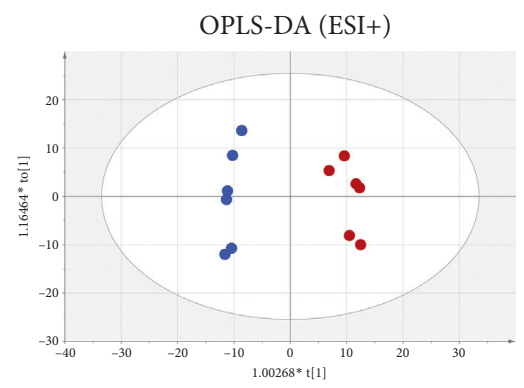

- ${ }^{\text {Model }} .52 \mathrm{~g} / \mathrm{kg}$ ZJP

(d)

OPLS-DA (ESI-)

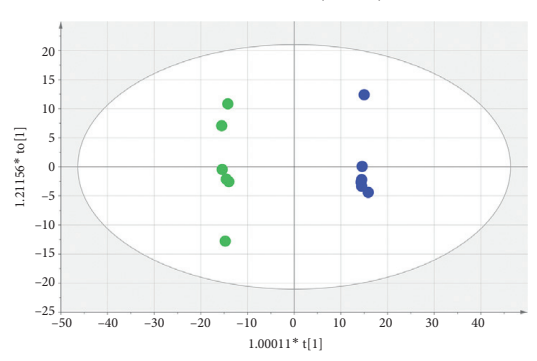

- Control

(g)

OPLS-DA (ESI-)

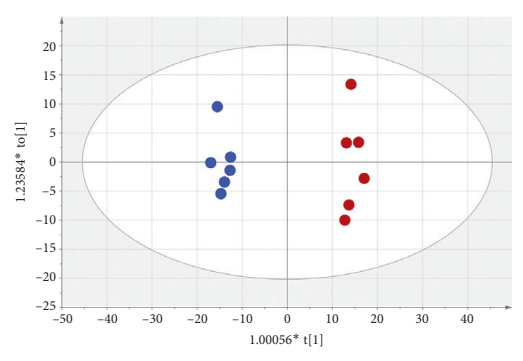

- $2.52 \mathrm{~g} / \mathrm{kg}$ ZJP

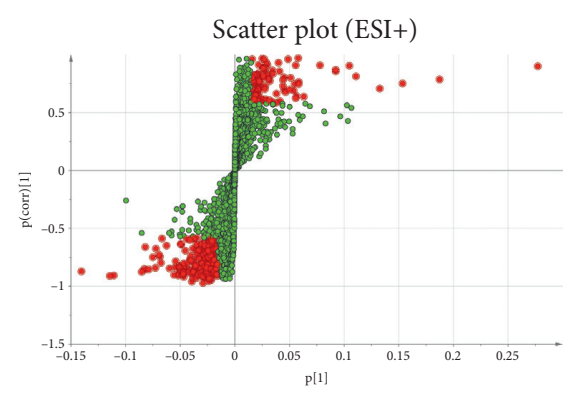

(b)

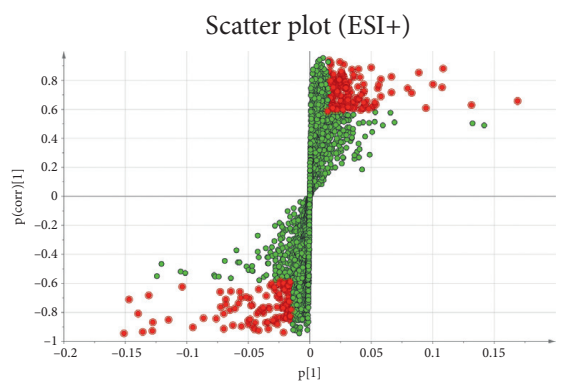

(e)

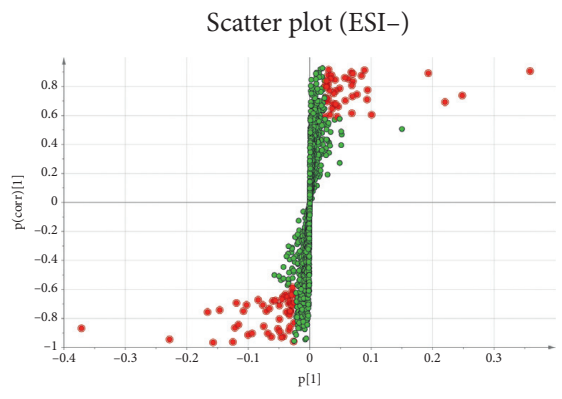

(h)

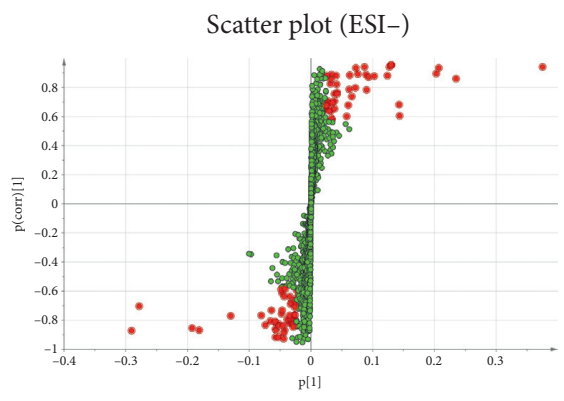

(k)

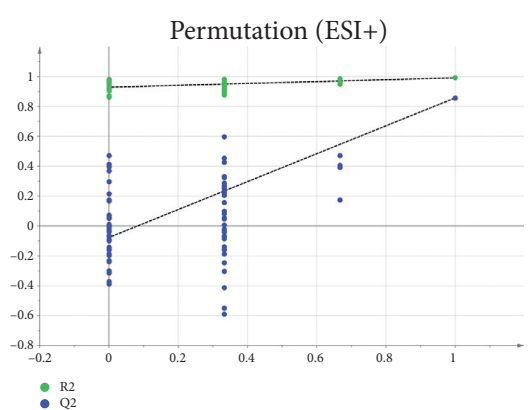

(c)

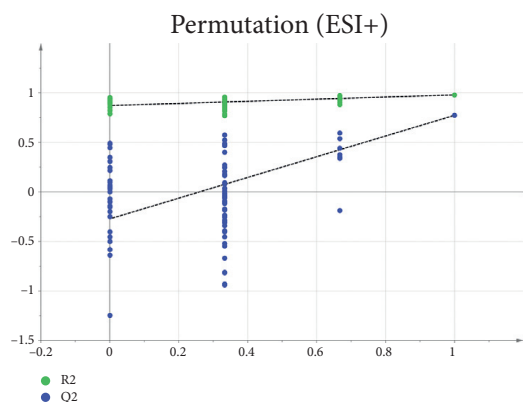

(f)

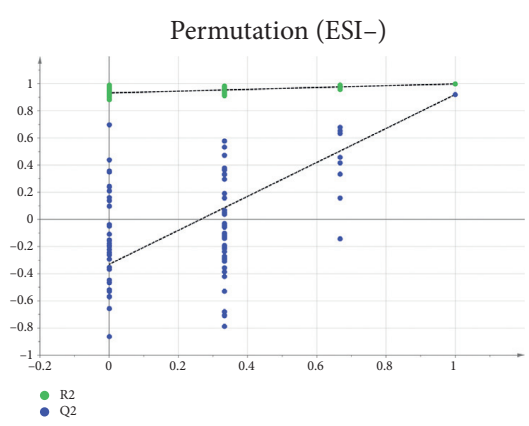

(i)

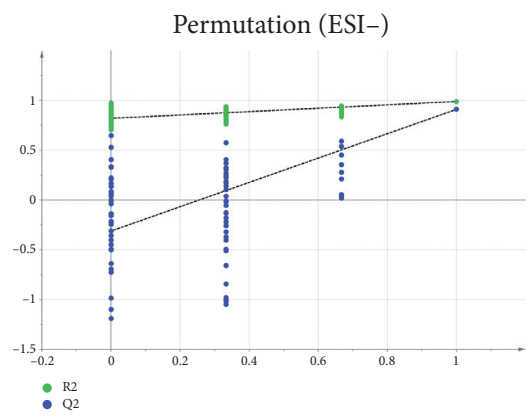

(l)

FIGURE 5: The OPLS-DA score plots, S-plot, and 100-permutation test generated of urine samples between the control group, model group, and $2.52 \mathrm{~g} / \mathrm{kg}$ ZJP group. OPLS-DA score plots were the pairwise comparisons in ESI+ mode, (a) control group versus model group and (d) model group versus $2.52 \mathrm{~g} / \mathrm{kg}$ ZJP group, as well as in ESI- mode, ( $\mathrm{g}$ ) control group versus model group and (j) model group versus $2.52 \mathrm{~g} / \mathrm{kg}$ ZJP group. S-plot of the OPLS-DA model in ESI+ mode, (b) control group versus model group and (e) model group versus $2.52 \mathrm{~g} / \mathrm{kg}$ ZJP group as well as in ESI- mode, (h) control group versus model group and (k) model group versus $2.52 \mathrm{~g} / \mathrm{kg} \mathrm{ZJP}$ group. The 100 -permutation test of the OPLS-DA model in ESI+ mode, (c) control group versus model group and (f) model group versus $2.52 \mathrm{~g} / \mathrm{kg}$ ZJP group as well as in ESI- mode, (i) control group versus model group and (l) model group versus $2.52 \mathrm{~g} / \mathrm{kg}$ ZJP group. 
TABLE 2: Identification of serum and urinary metabolites in different groups.

\begin{tabular}{|c|c|c|c|c|c|c|}
\hline Number & $\mathrm{RT}(\min )$ & Mass & Metabolites & Formula & KEGG ID & Pathway \\
\hline 1 & 17.65 & 104.1069 & Choline & $\mathrm{C}_{5} \mathrm{H}_{14} \mathrm{NO}$ & $\mathrm{C} 00114$ & Glycine, serine, and threonine metabolism \\
\hline 2 & 2.33 & 120.0658 & L-Threonine & $\mathrm{C}_{4} \mathrm{H}_{9} \mathrm{NO}_{3}$ & C00188 & Glycine, serine, and threonine metabolism \\
\hline 3 & 7.58 & 126.9998 & Hydroxypyruvic acid & $\mathrm{C}_{3} \mathrm{H}_{4} \mathrm{O}_{4}$ & $\mathrm{C} 00168$ & Glyoxylate and dicarboxylate metabolism \\
\hline 4 & 0.90 & 132.0771 & Creatine & $\mathrm{C}_{4} \mathrm{H}_{9} \mathrm{~N}_{3} \mathrm{O}_{2}$ & C00300 & Glycine, serine, and threonine metabolism \\
\hline 5 & 0.89 & 124.0073 & Taurine & $\mathrm{C}_{2} \mathrm{H}_{7} \mathrm{NO}_{3} \mathrm{~S}$ & $\mathrm{C} 00245$ & Taurine and hypotaurine metabolism \\
\hline 6 & 14.66 & 119.034 & Succinic acid & $\mathrm{C}_{4} \mathrm{H}_{6} \mathrm{O}_{4}$ & $\mathrm{C} 00042$ & TCA cycle \\
\hline 7 & 0.78 & 173.0087 & cis-Aconitic acid & $\mathrm{C}_{6} \mathrm{H}_{6} \mathrm{O}_{6}$ & C00417 & TCA cycle \\
\hline 8 & 1.22 & 215.0165 & Citric acid & $\mathrm{C}_{6} \mathrm{H}_{8} \mathrm{O}_{7}$ & $\mathrm{C} 00158$ & TCA cycle \\
\hline 9 & 0.79 & 101.0239 & Succinic acid semialdehyde & $\mathrm{C}_{4} \mathrm{H}_{6} \mathrm{O}_{3}$ & $\mathrm{C} 00232$ & Alanine, aspartate, and glutamate metabolism \\
\hline 10 & 0.87 & 167.0202 & Uric acid & $\mathrm{C}_{5} \mathrm{H}_{4} \mathrm{~N}_{4} \mathrm{O}_{3}$ & C00366 & Purine metabolism \\
\hline
\end{tabular}

metabolomics and urine metabolomics based on UPLC-QTOF/MS method, combined with network pharmacology, were used to clarify the therapeutic mechanism of ZJP on CAG.

H. pylori is one of the most common bacteria worldwide and an important risk factor for inducing gastric cancer. Numerous studies have shown that $H$. pylori can colonize and grow in an extremely acidic gastric environment and establish a persistent infection that reduces host immune function and leads to gastritis and gastric cancer [23]. Currently, $H$. pylori is widely used to establish rodent models of CAG [24]. Therefore, in this study, the CAG rat model was established by gavage of $H$. pylori. HE staining confirmed that ZJP and omeprazole were effective in reducing gastric mucosal damage, maintaining epithelial structural integrity, and improving monocyte and lymphocyte infiltration. Serum biochemical parameters showed that ZJP and omeprazole significantly reduced serum levels of IL- $1 \beta$, IL-6, IL10 , and iNOS, restored the integrity of the gastric mucosa, and effectively reduced the expression of inflammatory factors. The results confirm that ZJP shows therapeutic effects on CAG, which is consistent with clinical efficacy.

Next, the changes in serum metabolic profile and urine metabolic profile after ZJP treatment were measured to confirm that ZJP restores the normal structure of the gastric mucosa by restoring potential metabolites to normal levels. The screened biomarkers may become effective targets for disease prevention, diagnosis, and treatment [25]. The PCA analysis showed that the distribution of metabolites was significantly different between the control group, the model group, and the $2.52 \mathrm{~g} / \mathrm{kg}$ ZJP group. The distribution of potential metabolic biomarkers was identified in the OPLSDA analysis. There was a significant separation of biomarkers between the control group and the model group, indicating that $H$. pylori had a significant effect on the serum metabolomic profile and urine metabolomic profile. There were also remarkable differences between the $2.52 \mathrm{~g} / \mathrm{kg}$ ZJP group and the model group, suggesting that ZJP has therapeutic effects. Finally, six and four potential metabolites were screened in serum and urine samples, respectively, to reveal the mechanism of ZJP in the treatment of CAG. These metabolites interact in different ways and regulate the progression of the disease. The results showed that the occurrence and development of CAG are regulated by the changes of many physiological and pathological-related biomarkers, most of which affect each other. ZJP can regulate the expression of the ten potential metabolites, demonstrating that ZJP may treat CAG induced by $H$. pylori through multiple targets and pathways.

In this study, seven major metabolic pathways were screened through the identification of ten differential metabolites, including taurine and hypotaurine metabolism; glyoxylate and dicarboxylate metabolism; glycine, serine, and threonine metabolism; glycerophospholipid metabolism; TCA cycle, alanine, aspartate, and glutamate metabolism; butanoate metabolism; and purine metabolism. Taurine and hypotaurine metabolism are the important metabolic pathways with a variety of pharmacological activities, such as participation in eye-brain development, immune system, osmoregulation, and antioxidant and anti-inflammatory activities. Moreover, according to previous studies, it has a protective effect against oxidative stress-induced gastrointestinal damage [26]. Taurine is one of the most abundant free amino acids in animal tissues and plays an important role in a variety of important physiological processes. Consistent with the presentation of patients with gastritis [27], serum taurine levels are reduced after $H$. pylori infection, which may be related to the inflammatory response. In addition, metabolic pathway analysis identified several amino acid-related pathways in CAG, including the metabolism of glycine, serine, and threonine and the metabolism of alanine, aspartate, and glutamate. The results of this study showed that the expression of choline, creatine, and succinic acid semialdehyde associated with amino acid metabolism was elevated and L-threonine expression was decreased in the model group, and this trend was ameliorated by ZJP administration. The parietal cells of the stomach contain a large number of mitochondria [28]. The participation of glyoxylate and dicarboxylate metabolism further supports the involvement of ZJP in energy metabolism. Mitochondria provide energy to the cells through oxidative phosphorylation, which plays an important role in maintaining the steady-state and function of the gastric mucosa. ZJP further promotes hydroxypyruvate expression by restoring energy metabolism. Purine is an important component of nucleotides during cell proliferation, and therefore impaired purine metabolism is associated with the development of inflammation and cancer [29]. The expression of uric acid was elevated in CAG rats, which is consistent with a previous study [30]. The TCA cycle is the final metabolic pathway of carbohydrates, lipids, and amino acids and is the most important metabolic pathway for energy 


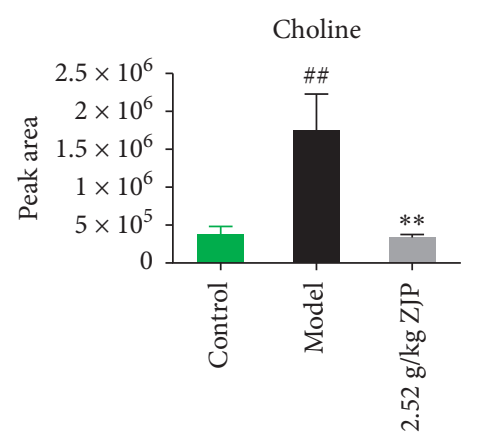

(a)

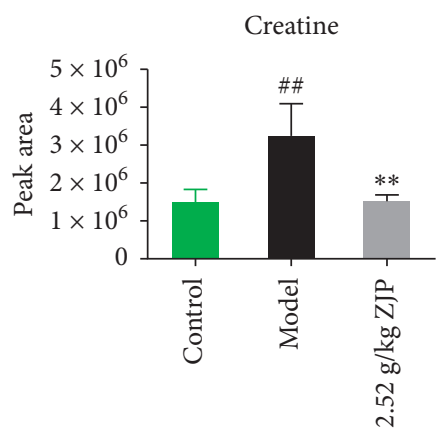

(d)

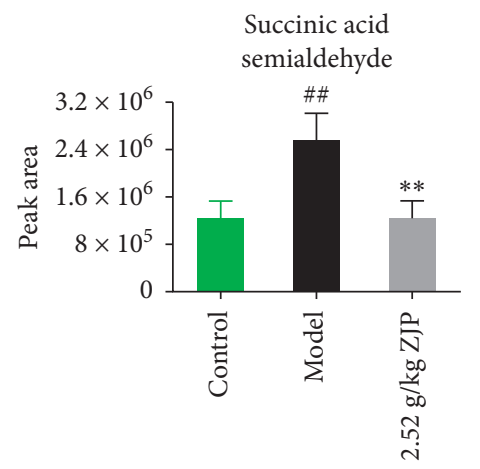

(g)

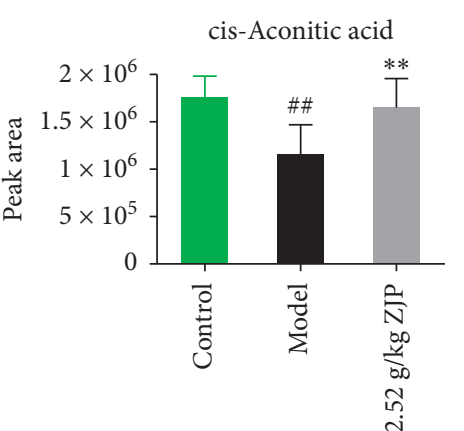

(b)

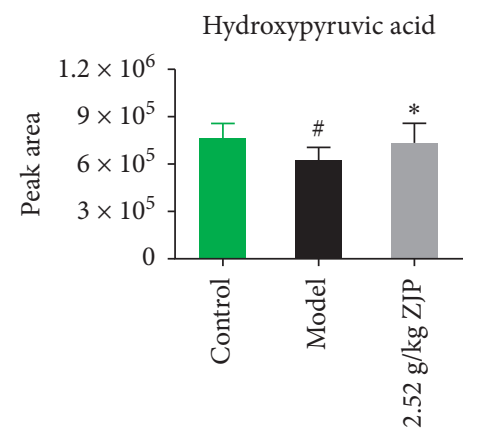

(e)

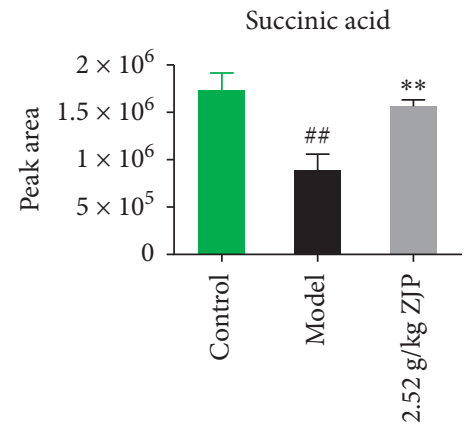

(h)

Uric acid

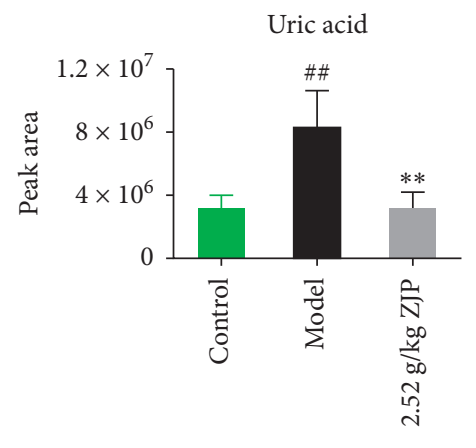

(j)

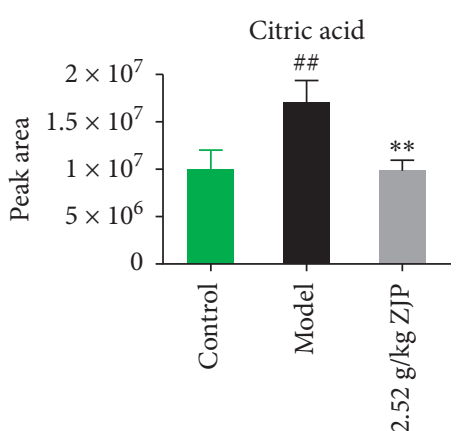

(c)

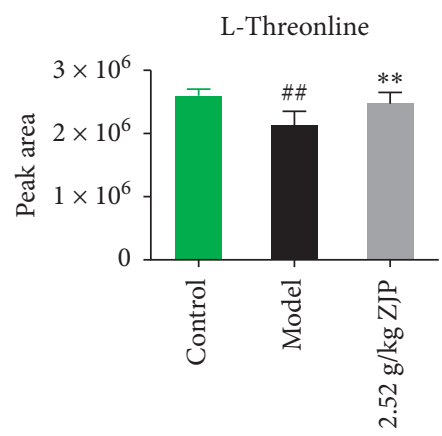

(f)

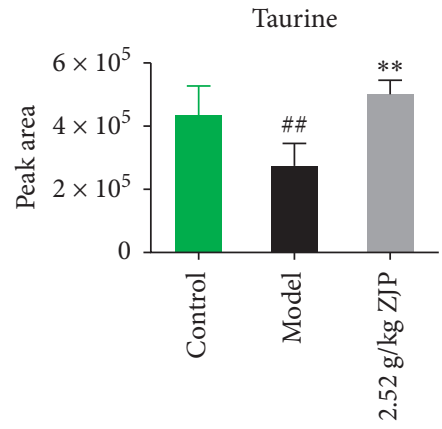

(i) 


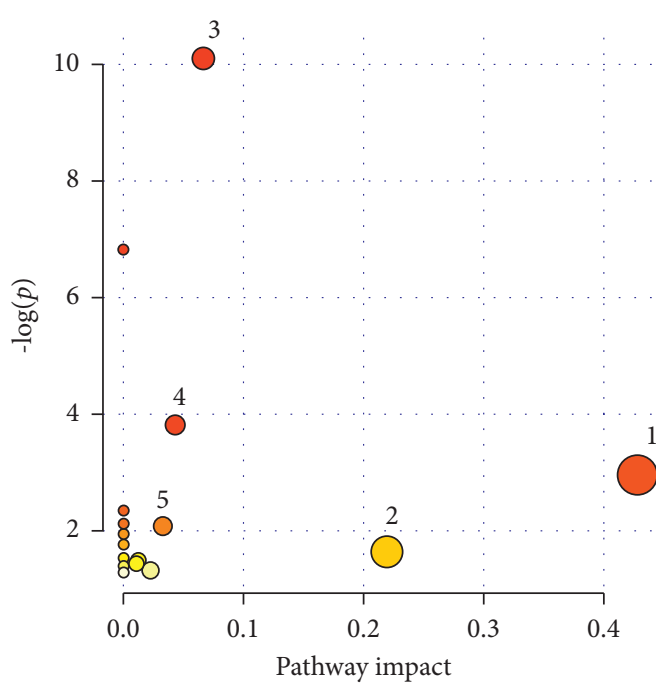

(a)

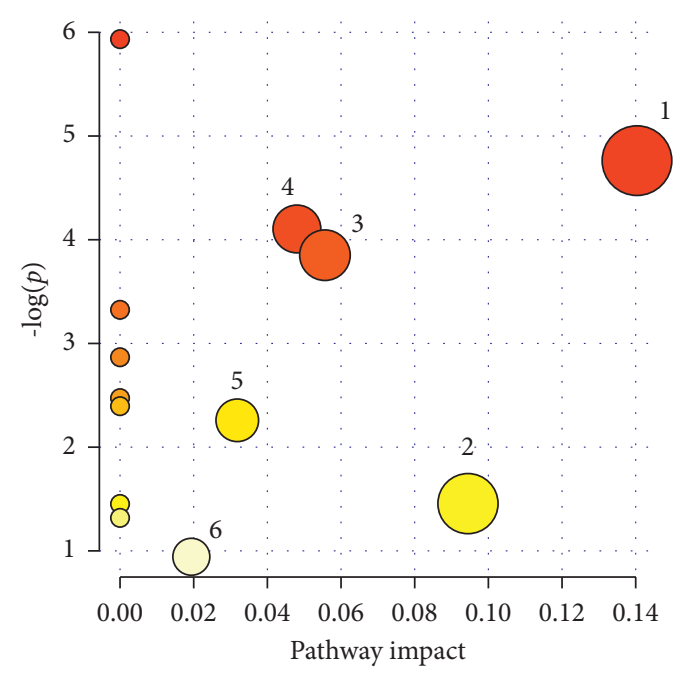

(b)

Figure 7: (a) Summary of metabolic pathway analysis of potential metabolites in serum samples. (1) Taurine and hypotaurine metabolism. (2) Glyoxylate and dicarboxylate metabolism. (3) Glycine, serine, and threonine metabolism. (4) Glycerophospholipid metabolism. (5) TCA cycle. (b) Summary of metabolic pathway analysis of potential metabolites in urine samples. (1) TCA cycle. (2) Glycerophospholipid metabolism. (3) Glyoxylate and dicarboxylate metabolism. (4) Alanine, aspartate, and glutamate metabolism. (5) Butanoate metabolism. (6) Purine metabolism.

TABLE 3: Results of integrating enrichment analysis of biomarkers with MetaboAnalyst.

\begin{tabular}{ccccccc}
\hline & Number & Pathway name & Match status & $P$ value & $-\log (P)$ & Impact \\
\hline \multirow{5}{*}{ Serum } & 1 & Taurine and hypotaurine metabolism & $1 / 8$ & 0.05192 & 2.9581 & 0.42857 \\
& 2 & Glyoxylate and dicarboxylate metabolism & $1 / 32$ & 0.19345 & 1.6427 & 0.21958 \\
& 3 & Glycine, serine, and threonine metabolism & $4 / 34$ & $4.1098 \times 10^{-5}$ & 10.1 & 0.06662 \\
& 4 & Glycerophospholipid metabolism & $2 / 36$ & 0.022085 & 3.8128 & 0.04318 \\
& 5 & Citrate cycle (TCA cycle) & $1 / 20$ & 0.12526 & 2.0774 & 0.03273 \\
\hline \multirow{5}{*}{ Urine } & 6 & Citrate cycle (TCA cycle) & $2 / 20$ & 0.0085481 & 4.762 & 0.14041 \\
& 7 & Glycerophospholipid metabolism & $1 / 36$ & 0.23395 & 1.4527 & 0.09446 \\
& 8 & Glyoxylate and dicarboxylate metabolism & $2 / 32$ & 0.021272 & 3.8503 & 0.05556 \\
& 9 & Alanine, aspartate, and glutamate metabolism & $2 / 28$ & 0.016472 & 4.1061 & 0.04808 \\
& 10 & Butanoate metabolism & $1 / 15$ & 0.1044 & 0.1044 & 0.03175 \\
& 11 & Purine metabolism & $1 / 66$ & 0.38959 & 0.94266 & 0.94266 \\
\hline
\end{tabular}

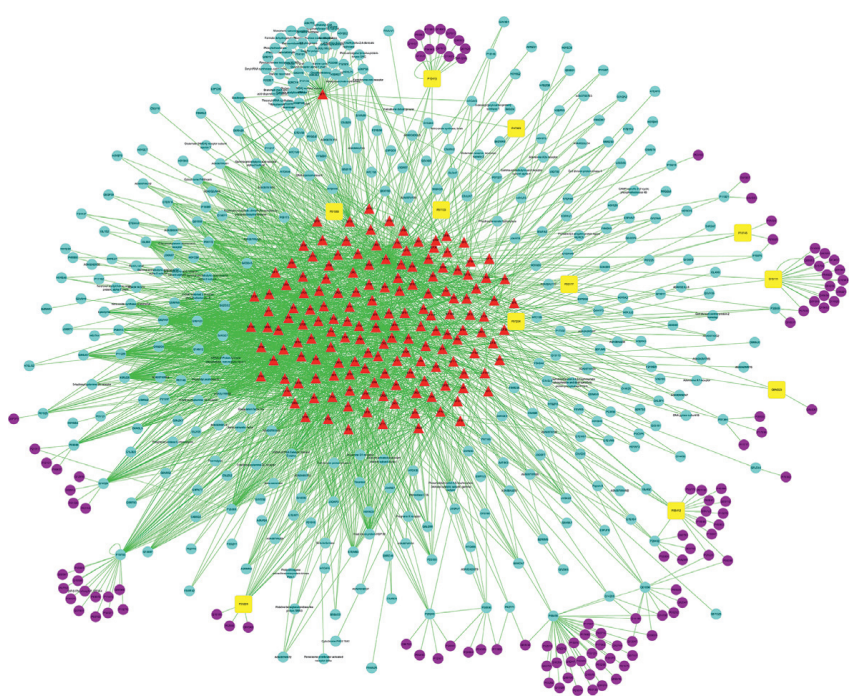

(a)

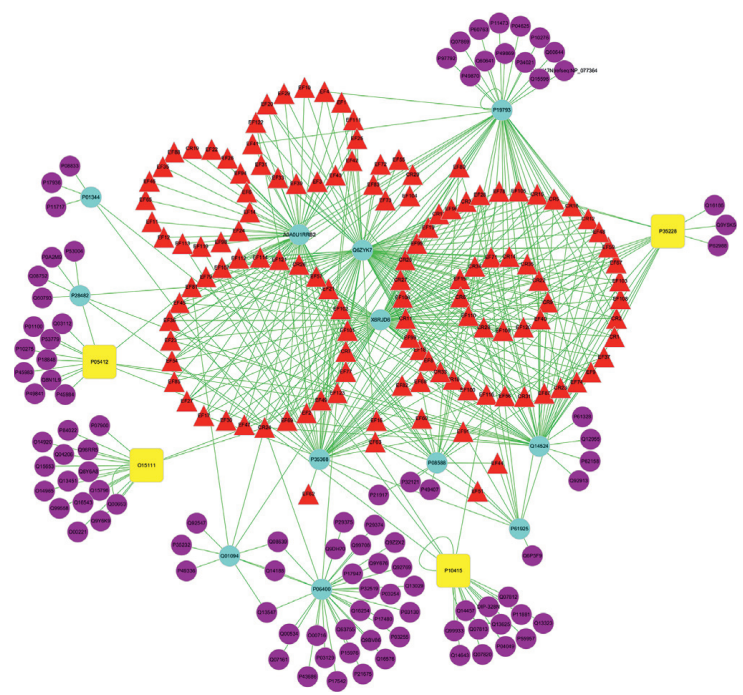

(b)

FIgURE 8: The crucial compound-CAG target-metabolite network. (a) All target information participating in the treatment of CAG by ZJP. (b) Key target information participating in the treatment of CAG by ZJP. The red triangle represents the active chemical composition of ZJP; the blue dots represent the protein targets of ZJP; the yellow square represents the common target for ZJP and disease; and the purple dots represent targets associated with potential metabolites. 
Table 4: Participating in the "Component-Target-Potential Metabolite" interactive network for ZJP treatment of CAG.

\begin{tabular}{lcc}
\hline Uniprot ID & Gene name & Protein name \\
\hline P28482 & MAPK1 & Mitogen-activated protein kinase 1 \\
Q6ZYK7 & PTGS2 & Prostaglandin G/H synthase 2 \\
X6RJD6 & PTGS1 & Prostaglandin G/H synthase 1 \\
P19793 & RXRA & Retinoic acid receptor RXR-alpha \\
P35368 & ADRA1B & Alpha-1B adrenergic receptor \\
P06400 & RB1 & Retinoblastoma-associated protein \\
P08588 & ADRB1 & Beta-1 adrenergic receptor \\
Q01094 & E2F1 & Transcription factor E2F1 \\
Q14524 & SCN5A & Sodium channel protein type 5 subunit alpha \\
P61925 & PKIA & cAMP-dependent protein kinase inhibitor alpha \\
P01344 & IGF2 & Insulin-like growth factor II \\
A0A0U1RRB2 & GABRA1 & Gamma-aminobutyric acid receptor subunit alpha-1 \\
\hline
\end{tabular}

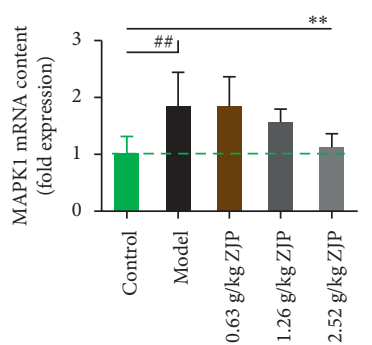

(a)

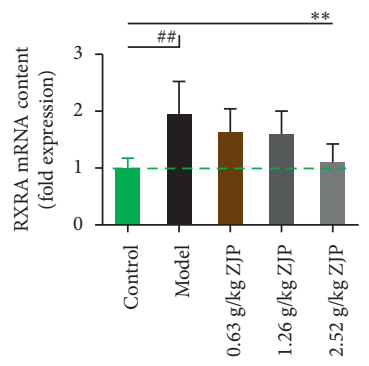

(e)

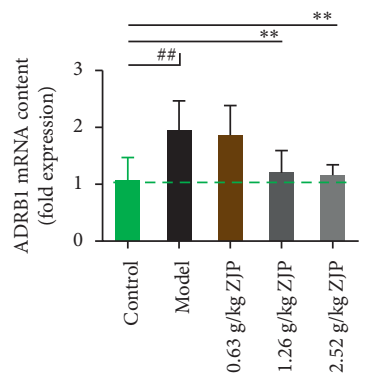

(i)

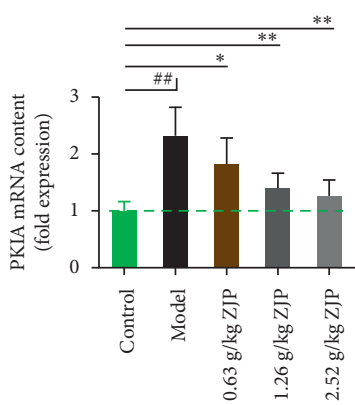

(b)

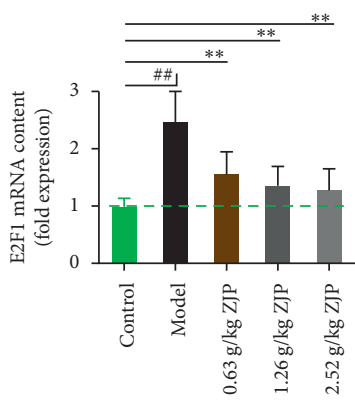

(f)

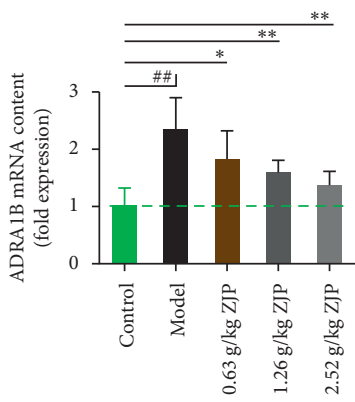

(j)

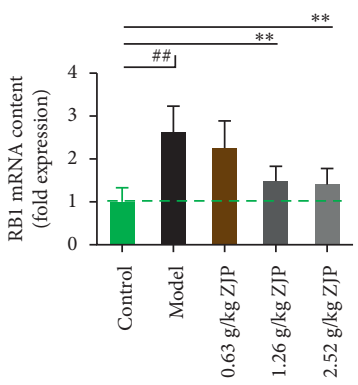

(c)

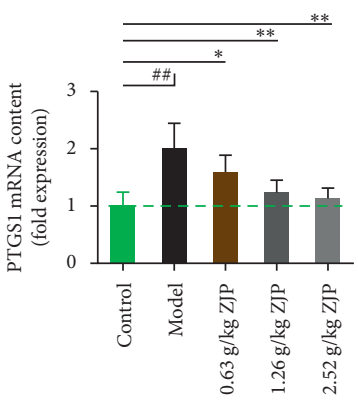

(g)

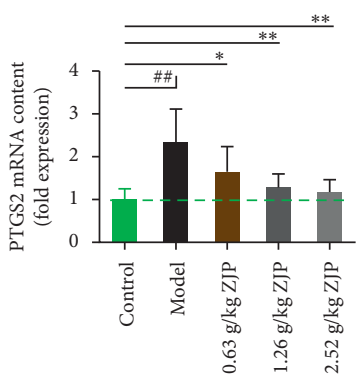

$(\mathrm{k})$

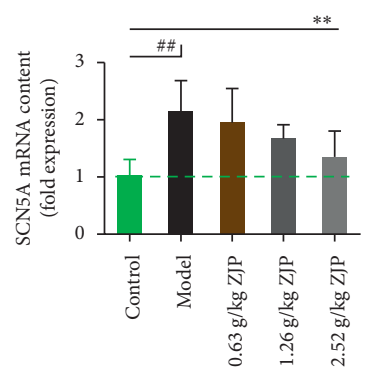

(d)

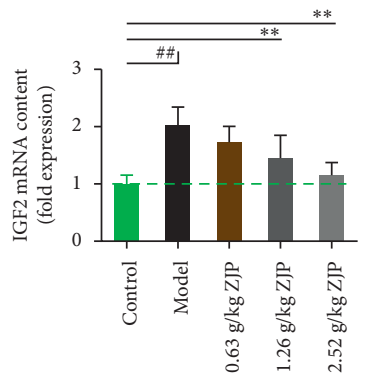

(h)

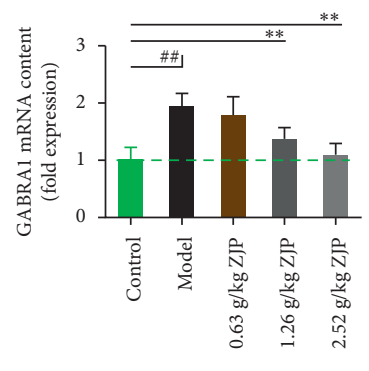

(1)

Figure 9: Effect of ZJP on the relative mRNA expressions of MAPK1, PKIA, RB1, SCN5A, RXRA, E2F1, PTGS1, IGF2, ADRB1, ADRA1B, PTGS2, and GABRA1 in CAG rats. (a) MAPK1; (b) PKIA; (c) RB1; (d) SCN5A; (e) RXRA; (f) E2F1; (g) PTGS1; (h) IGF2; (i) ADRB1; (j) ADRA1B; (k) PTGS2; (l) GABRA1. ${ }^{\# \#} P<0.01$, compared with the control group. ${ }^{*} P<0.05$ and ${ }^{* *} P<0.01$, compared with the model group.

To further understand the mechanism of ZJP treatment and the relationship between chemical components and metabolites, we combined network pharmacology to establish a "compounds-disease targets-metabolites" interaction network. The results showed that 124 chemical components from ZJP directly acted on 12 potential protein targets, indicating that multiple components in ZJP are involved in regulating endogenous metabolic changes and 
the advantages of ZJP in the clinical treatment of CAG. Twelve targets are closely associated with inflammation, including MAPK1, PTGS2, PTGS1, RXRA, ADRA1B, RB1, ADRB1, E2F1, SCN5A, PKIAIGF2, and GABRA1 [33-36]. Therefore, in order to verify the accuracy of network pharmacology prediction and clarify the underlying mechanism of ZJP, we further tested the expression of these genes in gastric tissue. The expression of MAPK1, PTGS2, PTGS1, RXRA, ADRA1B, RB1, ADRB1, E2F1, SCN5A, PKIAIGF2, and GABRA1 was significantly elevated in the model group. However, ZJP administration significantly reduced the relative mRNA expression levels of these genes. The results showed that ZJP could exert a protective effect through anti-inflammation, which plays an important role in the treatment of CAG.

\section{Conclusion}

This study is the first to systematically investigate the efficacy and molecular mechanisms of ZJP in the treatment of $H$. pylori-induced CAG through the combination of metabolomics and network pharmacology. The results showed that a series of inflammatory markers, such as MAPK1, PKIA, RB1, SCN5A, RXRA, E2F1, PTGS1, IGF2, ADRB1, ADRA1B, PTGS2, and GABRA1, played a dominant role in the ZJP treatment. The screened metabolic markers and metabolic pathways may serve as potential drug targets for the diagnosis and treatment of CAG, which may lay the foundation for further exploration of the interactions between herbal components and targets and provide a theoretical basis for guiding the clinical practice of TCM.

\section{Data Availability}

The figure data and related data used to support the findings of this study are included within the article.

\section{Ethical Approval}

This study was carried out with review and approval from the Animal Ethical and Experimental Committee of the Fifth Medical Center of PLA General Hospital (ID: IACUC-2018010).

\section{Conflicts of Interest}

The authors declare that they have no conflicts of interest.

\section{Authors' Contributions}

HL, RW, and JL designed the study; SW, XC, and MW conducted experiment and data analysis; JW, TY, YW, SR, SW, MJ, HL, and HX drafted the manuscript; and YZ supervised the study and provided funding acquisition. All the authors participated in amending the manuscript before submission of the mutually agreed final version.

\section{Acknowledgments}

This work was financially supported by the National Key R\&D Program of China (no. 2018YFC1704500). The authors acknowledge Research Square.

\section{References}

[1] The EUROGAST Study Group, "An international association between Helicobacter pylori infection and gastric cancer," Lancet, vol. 341, no. 8857, pp. 1359-1362, 1993.

[2] N. Uemura, S. Okamoto, S. Yamamoto et al., "Helicobacter pyloriInfection and the development of gastric cancer," New England Journal of Medicine, vol. 345, no. 11, pp. 784-789, 2001.

[3] H. Yoon, N. Kim, B. H. Lee et al., "Moxifloxacin-containing triple therapy as second-line treatment for Helicobacter pylori infection: effect of treatment duration and antibiotic resistance on the eradication rate," Helicobacter, vol. 14, no. 5, pp. 77-85, 2009.

[4] B.-L. Ma and Y.-M. Ma, "Pharmacokinetic properties, potential herb-drug interactions and acute toxicity of oralRhizoma coptidisalkaloids," Expert Opinion on Drug Metabolism \& Toxicology, vol. 9, no. 1, pp. 51-61, 2013.

[5] Y. F. Chen, W. W. Chen, and R. L. Li, "Effect of Zuojin wan and retro-zuojin wan on inflammatory and protection factors of chills and fever gastric mucosa injury," Chinese Journal of Integrated Traditional and Western Medicine, vol. 11, pp. 133-135, 2003.

[6] D. H. Cheng, Y. L. Zhao, and H. B. Yang, "Zhongguo Zhong Xi Yi Jie He Za Zhi Zhongguo Zhongxiyi Jiehe Zazhi," Chinese Journal of Integrated Traditional and Western Medicine, vol. 31, no. 2, pp. 209-212, 2011.

[7] S. T. Chou, C. Y. Hsiang, H. Y. Lo et al., "Exploration of anticancer effects and mechanisms of Zuo-Jin-Wan and its alkaloid components in vitro and in orthotopic HepG2 xenograft immunocompetent mice," BMC Complementary and Alternative Medicine, vol. 17, no. 1, p. 121, 2017.

[8] J. Pan, Y. Xu, H. Song, X. Zhou, Z. Yao, and G. Ji, "Extracts of Zuo Jin Wan, a traditional Chinese medicine, phenocopies 5HTR1D antagonist in attenuating $\mathrm{Wnt} / \beta$-catenin signaling in colorectal cancer cells," BMC Complementary and Alternative Medicine, vol. 17, no. 1, p. 506, 2017.

[9] J. Wang, T. Zhang, L. Zhu, C. Ma, and S. Wang, "Anti-ulcerogenic effect of Zuojin Pill against ethanol-induced acute gastric lesion in animal models," Journal of Ethnopharmacology, vol. 173, pp. 459-467, 2015.

[10] T. Wang, Y.-F. Yan, L. Yang et al., "Effects of Zuojin pill on depressive behavior and gastrointestinal function in rats with chronic unpredictable mild stress: role of the brain-gut axis," Journal of Ethnopharmacology, vol. 254, p. 112713, 2020.

[11] Q.-S. Wang, Y.-L. Cui, T.-J. Dong, X.-F. Zhang, and K.-M. Lin, "Ethanol extract from a Chinese herbal formula, "Zuojin Pill", inhibit the expression of inflammatory mediators in lipopolysaccharide-stimulated RAW 264.7 mouse macrophages," Journal of Ethnopharmacology, vol. 141, no. 1, pp. 377-385, 2012.

[12] A. N. Zira, S. E. Theocharis, D. Mitropoulos, V. Migdalis, and E. Mikros, "1H NMR metabonomic analysis in renal cell carcinoma: a possible diagnostic tool," Journal of Proteome Research, vol. 9, no. 8, pp. 4038-4044, 2010.

[13] L. Wu, H. Hao, and G. Wang, "LC/MS based tools and strategies on qualitative and quantitative analysis of herbal 
components in complex matrixes," Current Drug Metabolism, vol. 13, no. 9, pp. 1251-1265, 2012.

[14] W. B. Dunn, D. Broadhurst, D. Broadhurst et al., "Procedures for large-scale metabolic profiling of serum and plasma using gas chromatography and liquid chromatography coupled to mass spectrometry," Nature Protocols, vol. 6, no. 7, pp. 1060-1083, 2011.

[15] Q.-J. Yang, J.-R. Zhao, J. Hao et al., "Serum and urine metabolomics study reveals a distinct diagnostic model for cancer cachexia," Journal of Cachexia, Sarcopenia and Muscle, vol. 9, no. 1, pp. 71-85, 2018.

[16] J.-l. Ren, H. Dong, Y. Han et al., "Network pharmacology combined with metabolomics approach to investigate the protective role and detoxification mechanism of Yunnan Baiyao formulation," Phytomedicine, vol. 77, p. 153266, 2020.

[17] K. Ning, X. Zhao, A. Poetsch, W. H. Chen, and J. Yang, "Computational molecular networks and network pharmacology," Biomed Research International, vol. 2017, Article ID 7573904, 1 page, 2017.

[18] A.-L. Barabási and Z. N. Oltvai, "Network biology: understanding the cell's functional organization," Nature Reviews Genetics, vol. 5, no. 2, pp. 101-113, 2004.

[19] H. Blasco, J. Błaszczyński, J. C. Billaut et al., "Comparative analysis of targeted metabolomics: dominance-based rough set approach versus orthogonal partial least square-discriminant analysis," Journal of Biomedical Informatics, vol. 53, pp. 291-299, 2015.

[20] B. Worley and R. Powers, "PCA as a practical indicator of OPLS-DA model reliability," Current Metabolomics, vol. 4, no. 2, pp. 97-103, 2016.

[21] S. Wei, L. Qian, M. Niu et al., "The modulatory properties of Li-Ru-kang treatment on hyperplasia of mammary glands using an integrated approach," Frontiers in Pharmacology, vol. 9, p. 651, 2018.

[22] Y. Zhang, J. Gao, Q. Zhang et al., "Interpretation of Euphorbia kansui stir-fried with vinegar treating malignant ascites by a UPLC-Q-TOF/MS based rat serum and urine metabolomics strategy coupled with network pharmacology," Molecules, vol. 23, no. 12, p. 3246, 2018.

[23] V. Camilo, T. Sugiyama, and E. Touati, "Pathogenesis of Helicobacter pylori infection," Helicobacter, vol. 22, no. 1, 2017.

[24] X. Wei, X.-P. Feng, L.-Y. Wang et al., "Improved method for inducing chronic atrophic gastritis in mice," World Journal of Gastrointestinal Oncology, vol. 11, no. 12, pp. 1115-1125, 2019.

[25] W. Jiang, L. Si, P. Li et al., "Serum metabonomics study on antidiabetic effects of fenugreek flavonoids in streptozotocininduced rats," Journal of Chromatography B, vol. 1092, pp. 466-472, 2018.

[26] J. Zhou, N. Yao, S. Wang et al., "Fructus Gardeniae-induced gastrointestinal injury was associated with the inflammatory response mediated by the disturbance of vitamin B6, phenylalanine, arachidonic acid, taurine and hypotaurine metabolism," Journal of Ethnopharmacology, vol. 235, pp. 47-55, 2019.

[27] A. W. Chan, R. S. Gill, D. Schiller, and M. B. Sawyer, "Potential role of metabolomics in diagnosis and surveillance of gastric cancer," World Journal of Gastroenterology, vol. 20, no. 36, pp. 12874-12882, 2014.

[28] J. G. Duman, N. J. Pathak, M. S. Ladinsky, K. L. McDonald, and J. G. Forte, "Three-dimensional reconstruction of cytoplasmic membrane networks in parietal cells," Journal of Cell Science, vol. 115, no. Pt 6, pp. 1251-1258, 2002.
[29] J. Yin, W. Ren, X. Huang, J. Deng, T. Li, and Y. Yin, "Potential mechanisms connecting purine metabolism and cancer therapy," Frontiers in Immunology, vol. 9, p. 1697, 2018.

[30] H. Zhang, P. Fu, B. Ke et al., "Metabolomic analysis of biochemical changes in the plasma and urine of collageninduced arthritis in rats after treatment with Huang-Lian-JieDu-Tang," Journal of Ethnopharmacology, vol. 154, no. 1, pp. 55-64, 2014.

[31] R. Shiratori, K. Furuichi, M. Yamaguchi et al., "Glycolytic suppression dramatically changes the intracellular metabolic profile of multiple cancer cell lines in a mitochondrial metabolism-dependent manner," Scientific Reports, vol. 9, no. 1, p. 18699, 2019.

[32] M. Chen, R. Liu, and Y. Weng, "Discovery and enlightenment of the tricarboxylic acid cycle," Philosophy of Medicine, vol. 33, no. 1, pp. 71-73, 2012.

[33] I. Bjarnason, C. Scarpignato, E. Holmgren, M. Olszewski, K. D. Rainsford, and A. Lanas, "Mechanisms of damage to the gastrointestinal tract from nonsteroidal anti-inflammatory drugs," Gastroenterology, vol. 154, no. 3, pp. 500-514, 2018.

[34] J. McAbee, Q. Li, H. Yu, and K. L. Kirkwood, "Sexual dimorphism in periapical inflammation and bone loss from mitogen-activated protein kinase phosphatase-1 deficient mice," Journal of Endodontics, vol. 38, no. 8, pp. 1097-1100, 2012.

[35] A. Mukwaya, A. Lennikov, M. Xeroudaki et al., "Time-dependent LXR/RXR pathway modulation characterizes capillary remodeling in inflammatory corneal neovascularization," Angiogenesis, vol. 21, no. 2, pp. 395-413, 2018.

[36] C. R. White, M. Dungan, and M. D. Carrithers, "Activation of human macrophage sodium channels regulates RNA processing to increase expression of the DNA repair protein PPP1R10," Immunobiology, vol. 224, no. 1, pp. 80-93, 2019. 